\title{
Resultado global y dimensión empresarial para cotizadas europeas en NYSE y NASDAQ
}

\author{
Sousa Fernández, Francisco* \\ Carro Arana, María Mercedes**
}

\section{Resumen}

El resultado global está cobrando cada vez más fuerza como referente del resultado empresarial frente al más tradicional resultado neto, en particular, desde la revisión en el año 2007 de la Norma Internacional de Contabilidad n ${ }^{\circ} 1$ (NIC 1) del Comité de Normas Internacionales de Contabilidad (IASB). Además, se está asistiendo al impacto del mismo a nivel internacional a través de la adopción de las normas de este organismo, como ocurre en Venezuela con los Boletines de Aplicación de las mismas, emitidos por la Federación de Colegios de Contadores Públicos. Ante este escenario, este trabajo pionero a nivel internacional, plantea como objetivo esencial evaluar empíricamente cómo impacta el resultado global en los grupos empresariales atendiendo a su dimensión, con especial atención a las grandes corporaciones por su reticencia a la adopción del mismo por temor a perjudicar sus resultados. Para ello, se toma una muestra de 136 grupos empresariales europeos cotizados en NYSE y NASDAQ con información revelada según normas norteamericanas. Como metodología de contraste se emplean herramientas no paramétricas al estar los datos muy alejados de la normalidad. En concreto, como pruebas a priori se utiliza el Test de Kruskal-Wallis reforzado con el de la Mediana y como pruebas a posteriori el Test de la $U$ de Mann-Whitney reforzado con el de KolmogorovSmirnov para dos muestras. Los resultados muestran un acusado impacto negativo del resultado global frente al resultado neto, en algunos casos espectacular, que conduce a diferencias significativas, en particular, entre los pequeños y grandes grupos empresariales, lo que puede ser de utilidad para la gerencia y el resto de usuarios.

Palabras clave: resultado global, resultado neto, dimensión empresarial, grupos europeos cotizados en mercados norteamericanos.

Recibido: 15-05-10. Aceptado: 07-12-10

* Doctor en Ciencias Económicas y Empresariales (Premio Extraordinario de Doctorado), Profesor e Investigador en Contabilidad, Universidad de Cantabria (España), autor para la correspondencia. e-mail: sousaf@unican.es.

** Doctora en Ciencias Económicas y Empresariales, Profesora e Investigadora en Contabilidad, Universidad de Cantabria (España), Auditora de Cuentas. e-mail: carrom@unican.es. 


\title{
Comprehensive Income and Company Size for European Groups Listed on the NYSE and NASDAQ
}

\begin{abstract}
Comprehensive income is becoming a stronger reference for measuring business performance compared to the more traditional use of net income, in particular since the 2007 review of International Accounting Standard 1 of the International Accounting Standards Board. Furthermore, its impact on an international level can be witnessed through the adoption of this body's Standards. This is the case in Venezuela with Application Bulletins for these Standards issued by the Federation of Public Accountant Associations. In such a scenario, this pioneering work, conducted at an international level, raises the essential objective of empirically assessing how comprehensive income impacts business groups according to their size, paying special attention to the reluctance of large corporations to adopt these measures for fear of damaging their results. For this analysis, a sample of 136 European business groups listed on the NYSE and NASDAQ was chosen according to information based on North American standards. A set of non-parametric tools was used to perform the corresponding statistical comparisons, since the data was quite far from normal. Specifically, the KruskalWallis Test reinforced with the Mean Test was used a priori; the Mann-Whitney U Test backed by the Kolmogorov-Smirnov Two-Sample Test was used a posteriori. Results show a marked, and in some cases dramatic, negative impact when comprehensive income was compared to net income, leading to significant differences, especially between small and large business groups, which may be useful for managers and other users.
\end{abstract}

Key words: comprehensive income, net income, business size, European groups listed on North American markets.

\section{Introducción}

Los organismos emisores de normas contables más relevantes a nivel internacional, entre los que cabe destacar el Comité de Normas de Contabilidad Financiera (FASB) en su Norma de Contabilidad Financiera 130 (SFAS 130, 1997) y el IASB con la NIC 1 (1997, revisada en 2003 y 2007); vienen exigiendo a las empresas la revelación del resultado global, concebido en el Marco Conceptual de la Contabilidad Financiera (SFAC 6, 1985, párr. 70) del FASB como todos los cambios en el patrimonio neto de la empresa en un de- terminado ejercicio económico, eliminando operaciones con los propietarios.

Asimismo, dada la tendencia a la adopción de las Normas Internacionales de Información Financiera (NIIF) del IASB (International Accounting Standard Board) a escala mundial, como ha ocurrido en Venezuela con los Boletines de Aplicación de dichas normas internacionales, emitidos por la Federación de Colegios de Contadores Públicos, en concreto, con el Boletín de Aplicación VENNIF nº (VA-BEN-NIF 0); el resultado global está cobrando cada vez más fuerza a nivel internacional como referente del 
rendimiento empresarial frente al más tradicional resultado neto.

Dicho resultado se determina agregando al resultado neto, que es la última magnitud de la Cuenta de Resultados tradicional, los gastos e ingresos que de acuerdo con las normas correspondientes deban reconocerse directamente en el patrimonio neto, como pueden ser los beneficios y pérdidas no realizados en los activos financieros clasificados a efectos de su valoración como disponibles para la venta, los ajustes asociados a las coberturas de flujos de efectivo y de una inversión neta en el exterior y las diferencias de cambio que surgen al convertir los activos y pasivos de las filiales en el proceso de consolidación. Por todo ello, se está ante un resultado contable más conectado con la realidad de los mercados que el más tradicional resultado neto.

En el marco de estas tendencias en la información financiera, este trabajo pionero a nivel internacional, previa revisión de la literatura, plantea como objetivo esencial evaluar empíricamente cómo impacta el resultado global en los grupos empresariales atendiendo a su dimensión, y así poder aportar evidencia con respecto a si se está ante un fenómeno que afecta de forma similar a los mismos, o si por el contrario se presentan diferencias significativas en función de su tamaño.

Para ello, se toma como referencia una muestra de 136 grupos empresariales europeos cotizados en la Bolsa de Nueva York (New York Stock Exchange -NYSE-) y en la Cotización Automatizada de la Asociación Nacional de Agentes de Valores (National Association of Secu- rities Dealers Automated Quotations -NASDAQ-), de acuerdo con la correspondiente información según el SFAS 130 del FASB revelada en el documento en formato $20-\mathrm{F}$ cuando presentaron dichos grupos las cuentas a la Comisión del Mercado de Valores (Securities and Exchange Commission-SEC-) los años del período 1999-2004.

Por otra parte, debido a que los datos sobre los que se construirá el estudio empírico se encuentran muy alejados de la normalidad, se emplearán como herramientas de contraste estadístico un conjunto de pruebas no paramétricas.

Se puede anticipar que se está ante un impacto negativo muy acusado del resultado global frente al resultado neto, que conduce a diferencias estadísticamente significativas, en particular, entre las empresas de pequeña y gran dimensión, lo que puede ser un referente para la toma de decisiones tanto por parte de la gerencia como del resto de interesados en la empresa.

Se seleccionó la muestra de 136 grupos empresariales europeos cotizados en NYSE y NASDAQ, en principio, por ser los primeros del continente europeo que han tenido que informar del resultado global de acuerdo con el SFAS 130 del FASB en las reconciliaciones con los Principios de Contabilidad Generalmente Aceptados en Estados Unidos (US GAAP) contenidas en los 20-F de la SEC.

Además, muchos de los grupos de la muestra forman parte en la actualidad del selectivo índice de la eurozona EURO STOXX 50, con lo que este estudio incluye algunos de los grupos empresariales 
de más relevancia de la zona del euro, y por ello, se está ante empresas con presencia mundial.

Asimismo, aunque haya algunas diferencias cuantitativas entre el resultado global que deben revelar las empresas estadounidenses de acuerdo con el SFAS 130 del FASB, y el que deben informar las empresas del resto del mundo que tengan que aplicar las NIIF del IASB, en particular, la NIC 1, se está ante un resultado contable sustentado sobre la misma base conceptual.

Por ello, las evidencias que se encuentren en la investigación pueden considerarse a modo de anticipo para cualquier entorno económico y contable, mucho más allá de las fronteras de cualquier país, de lo que ya está siendo y será la aplicación del resultado global a escala prácticamente universal con la adopción de las NIIF del IASB.

Por otra parte, es preciso señalar que se ha elegido el período de estudio 1999-2004 para con ello incluir el mayor número posible de grupos empresariales en la muestra. En este sentido, aunque el SFAS 130 del FASB fue aprobado en 1997, y ya se contaba en el año 1998 con información sobre el resultado global y también para 1997 como información comparativa, el haber incluido estos dos años hubiese supuesto la exclusión de determinados grupos empresariales dado que no cotizaron dichos años en NYSE o NASDAQ, por lo que no se dispone de información en la SEC.

Además, desde el primero de enero de 2005 las cotizadas de la Unión Europea deben de aplicar las NIIF del IASB para la formulación de los estados financieros consolidados. De acuerdo con di- chas normas, en concreto, conforme a la NIC 1 revisada en 2003, ya derogada, se exigía la revelación del resultado global bajo la particular denominación de "total ingresos y gastos reconocidos". Algunas empresas cotizadas en NYSE y NASDAQ a partir del 2005 fueron dejando de informar del resultado global conforme al SFAS 130 del FASB por considerar que coincidía con el de la NIC 1, pero como ya se ha referido no se da coincidencia plena entre ambos.

Por esa falta de homogeneidad, el haber considerado los años 2005 y siguientes en esta investigación, hubiera derivado en la exclusión de empresas, con una notable reducción de la muestra, por lo que se decidió configurar la misma hasta con datos del año 2004.

En cualquier caso, el período considerado 1999-2004, integra las fases de un ciclo económico, muy parejo al bursátil, que se caracterizó por la expansión de finales de los noventa, las fuertes caídas bursátiles de principios de los dos mil, en particular las del 2002, y la posterior recuperación de los años 2003 y 2004.

Estas circunstancias, el hecho de trabajar con una muestra de 136 grupos empresariales del continente europeo cotizados en NYSE y NASDAQ, muchos de ellos con presencial mundial, unido al objetivo que plantea este artículo de investigar cómo incide un nuevo concepto de resultado a nivel universal, el resultado global, en función de la dimensión empresarial; se considera que dota a este trabajo del suficiente interés y actualidad tanto para quienes tienen que tomar las decisiones gerenciales como para el resto de usuarios de la información financiera. 


\section{Fundamentos conceptuales del resultado global}

EI FASB fue el organismo pionero en incorporar el concepto de resultado global en su Marco Conceptual para la Contabilidad Financiera [SFAC 3 (1980), reemplazado por el SFAC 6 (1985)], donde en su párrafo 70 define el mismo como "los cambios en el patrimonio neto de una empresa de negocios durante el ejercicio económico, derivados de transacciones y otros eventos y circunstancias que no se correspondan con operaciones con los propietarios. Incluye todos los cambios en el patrimonio neto durante un período, excepto aquellos que provengan de inversiones o retiradas de fondos por los propietarios".

Como se podrá comprobar, se está próximo al concepto de renta, esgrimido por el Premio Nobel británico Hicks (1939: 172), cuando en su obra Value and Capital establece que el propósito del cálculo del beneficio es revelar cuánto puede consumir un individuo sin llegar a empobrecerse, para lo que formuló el siguiente concepto central de renta: "La renta de un hombre ( $\mathrm{sic}$ ) es el máximo valor que puede consumir durante un período y esperar estar igual de bien al final que al principio de dicho período".

Al trasladar este concepto al ámbito contable, de acuerdo con Alexander (1950: 15), se puede precisar el beneficio de la empresa de negocios como la cifra de dividendos que ésta puede repartir a sus propietarios sin menoscabar el capital invertido, esto es, manteniéndose en la misma situación de riqueza al final que al principio de un determinado ejercicio económico.
Esta conceptuación, evidentemente lleva a posicionarse en la teoría del excedente limpio -clean surplus- (Brief y Peasnell, 1996; Felthan y Ohlson, 1995; Beale y Davey 2000; y Mattessich, 2002; entre otros), conforme a la cual el resultado contable se determina comparando el valor en libros del neto patrimonial al final de un ejercicio económico con el registrado al inicio del mismo, una vez eliminadas las operaciones con los propietarios.

En definitiva, tal como refiere Sousa (2009a: 233), con la adopción del resultado global se está ante un acontecimiento destacado para la Contabilidad ahora a los inicios del siglo XXI, como es el acercamiento al concepto de resultado económico -economic concept of income- (Linsmeier et al., 1997: 123), propugnado por los autores de la escuela clásica normativo deductiva hace ya varias décadas (MacNeal, 1939; Edwards y Bell, 1961; Alexander, 1950; Moonitz, 1961 y Sprouse y Moonitz, 1962, entre otros).

Sin embargo, ahora no es concebido como una magnitud a priori única e incontestable como pretendían dichos autores, sino pensado para satisfacer las necesidades de los usuarios, en particular las de los inversores, dado que con el mismo se contribuye al funcionamiento eficiente del mercado y a la utilidad de la información contable para la valoración de las empresas en los mercados (Mora, 2004: 10).

Por otra parte se observa al revisar la literatura empírica sobre el resultado global en el panorama internacional de los últimos años, se observa que un conjunto de estudios de naturaleza descriptiva, se ocuparon de analizar cómo incide 
el resultado global en las empresas frente al más tradicional resultado neto (Luecke y Meeting, 1998; Bhamornsiri y Wiggins, 2001; Pandit et al., 2006; entre otros).

Asimismo, otro conjunto de trabajos se orientaron al mercado de capitales (Dhaliwal et al., 1999; O'Hanlon y Pope, 1999; Hodder et al., 2006, entre otros), pretendiendo evidenciar en líneas generales si el resultado global explica mejor que el resultado neto los rendimientos de las acciones.

Y otros analizaron el impacto del resultado global frente al resultado neto desde distintas ópticas [Sousa (2008 y 2009b) y Sousa and Carro (2009a, 2009b y 2009c)]; mientras que algunas investigaciones se orientaron al estudio del impacto en los analistas e inversores de los formatos para su presentación (Hirst y Hopkins, 1998; Maines y McDaniel, 2000; y Hunton et al., 2006).

Sin embargo, no se dispone de trabajos que hayan investigado una problemática con el objetivo, la metodología y la muestra como la que se plantean en este estudio. Por ello, se está ante un trabajo pionero a nivel internacional. Además, en estos tiempos en los que se está generalizando a nivel mundial la adopción de las NIIF del IASB, lo que lleva implícito la asunción del resultado global, confiere a esta investigación interés y actualidad a nivel mundial.

\section{Diseño de la investigación empírica}

Para perfilar la muestra se ha partido del listado de todas las cotizadas del continente europeo en NYSE y NASDAQ a 31 de diciembre de 2004, dado que di- chas empresas, desde la aprobación del SFAS 130 del FASB en el año 1997, están obligadas a revelar el resultado global en las reconciliaciones con los US GAAP en los documentos 20-F de la SEC.

Tal como se ha señalado, se ha limitado el período de estudio a los años 1999-2004 para con ello incluir el mayor número posible de grupos empresariales en la muestra. Así, el haber elegido un período más extenso hubiera mermado de forma notable el número de empresas por las razones que se han dado, con la consiguiente falta de representatividad del estudio.

En cualquier caso, también como ha quedado apuntado, dicho periodo de seis años resulta bastante adecuado a este estudio, ya que integra las fases de un ciclo económico, muy parejo al bursátil, caracterizado por la expansión de finales de los noventa, las fuertes caídas bursátiles en los inicios de los dos mil, en particular en el año 2002, y la recuperación de los años posteriores.

Por otra parte, con el propósito de homogeneizar la información se han tenido que aplicar los correspondientes filtros, lo que llevó a excluir determinados grupos empresariales europeos de entre los listados a 31 de diciembre de 2004 en NYSE y NASDAQ.

Las razones para la exclusión de los mismos fueron principalmente el no cotizar todos los años del período 19992004, no mostrar el resultado global de forma clara, o formular los estados financieros en fecha distinta del 31 de diciembre como hicieron bastantes grupos empresariales británicos. Se ha de tener en cuenta que el resultado global incorpora elementos vinculados a los precios de los 
títulos y a diferencias de cambio, por lo que se hace necesario contar con la misma fecha de cierre del ejercicio contable para todos los grupos empresariales, ya que en otro caso, supondría una distorsión en las comparaciones.

Después de hechas todas estas consideraciones, se llega a una muestra de 136 grupos empresariales, correspondientes a 19 países europeos, tal como se detalla en la Tabla 1, que representan un $56 \%$ del total de grupos empresariales del continente Europeo cotizados en NYSE y NASDAQ a 31 de diciembre de 2004.

Por lo tanto, se está ante una muestra con una representatividad elevada de los grupos empresariales europeos cotizados en NYSE y NASDAQ. Además, muchos de los que integran la muestra

\section{Tabla 1}

\section{Distribución de grupos empresariales de la muestra por países}

\begin{tabular}{|c|c|c|}
\hline País & $\begin{array}{l}\text { Número de grupos } \\
\text { empresariales } \\
\text { de la muestra }\end{array}$ & $\begin{array}{l}\text { Porcentaje de grupos empresariales de la muestra sobre el } \\
\text { total de grupos europeos cotizados en NYSE y NASDAQ a } \\
\qquad 31 \text { de diciembre de } 2004\end{array}$ \\
\hline Alemania & 10 & $59 \%$ \\
\hline Austria & 1 & $100 \%$ \\
\hline Bélgica & 2 & $100 \%$ \\
\hline Dinamarca & 1 & $25 \%$ \\
\hline España & 7 & $89 \%$ \\
\hline Finlandia & 3 & $75 \%$ \\
\hline Francia & 20 & $71 \%$ \\
\hline Grecia & 4 & $67 \%$ \\
\hline Hungria & 1 & $100 \%$ \\
\hline Irlanda & 5 & $45 \%$ \\
\hline Italia & 9 & $90 \%$ \\
\hline Luxemburgo & 4 & $67 \%$ \\
\hline Noruega * & 4 & $67 \%$ \\
\hline Países Bajos & 24 & $73 \%$ \\
\hline Portugal & 2 & $100 \%$ \\
\hline Reino Unido & 23 & $30 \%$ \\
\hline Suecia & 3 & $60 \%$ \\
\hline Suiza * & 12 & $80 \%$ \\
\hline Turquía * & 1 & $100 \%$ \\
\hline Total & 136 & $56 \%$ \\
\hline
\end{tabular}

* Estos países no pertenecen a la Unión Europea.

Fuente: elaboración propia, a partir de las cotizadas europeas en NYSE y NASDAQ y los 20-F de las SEC. 
forman parte en la actualidad del selectivo índice de la eurozona EURO STOXX 50 , con lo que este estudio incluye grupos empresariales de relevancia en la zona del euro.

Una vez que ha quedado perfilada la muestra, se toman en las reconciliaciones con los US GAAP en los 20-F de la SEC, para cada año del período 19992004 y grupo empresarial, el resultado global y el resultado neto de acuerdo con el SFAS 130 del FASB, referidos al cierre de cada ejercicio, expresados en millones de euros. En el caso de revelar información en moneda distinta de euro, se ha hecho la conversión a euros aplicando el tipo de cambio oficial vigente al cierre del ejercicio correspondiente.

Por otra parte, como el objetivo es estudiar cómo impacta el resultado global frente al más tradicional resultado neto teniendo en cuenta la dimensión de los grupos empresariales, obviamente se requiere perfilar la variable tamaño, lo que se hace considerando una variable no financiera como es el número de empleados.

Se adoptó como referente de la dimensión a los empleados porque tanto la cifra de negocio como el patrimonio neto, como resulta obvio, están relacionadas estrechamente con el resultado neto y el resultado global que lo engloba, sobre los cuales se construirá la variable a contrastar, y en caso de elegir las mismas como variables de tamaño cuando se practi- quen los contrastes asociados a la dimensión, se podría estar ante una interrelación directa o contaminación entre la referida variable a contrastar y el criterio seguido para clasificar las empresas por tamaño.

Asimismo, con respecto al total de activos, se ha decidido no considerar esta variable debido a que no se contaba para todos los grupos con esta magnitud referida a los mismos GAAP, y las discrepancias cuantitativas podrían resultar significativas de unos GAAP a otros, con la consiguiente distorsión en el análisis.

Con respecto a los empleados de los grupos, se ha tomado con carácter general el número de éstos a 31 de diciembre de cada año, y en algunos casos concretos la media de empleados de todo el ejercicio ${ }^{1}$.

A partir de estos datos se ha hecho la media de los empleados del período de estudio 1999-2004, para después ser categorizados por niveles de acuerdo con la herramienta que proporciona SPSS v.17 de asignación de rangos a casos con Ntles igual a tres, según se recoge en la Tabla 2.

Se quiere destacar que al nivel 1 quedan adscritos, entre otros, veintisiete grupos empresariales con menos de mil empleados. Al estar ante grupos europeos que también cotizan en mercados de valores norteamericanos, podría llamar la atención el hecho de contar con un conjunto de los mismos de tan reducida cierre o de la media registrada en el ejercicio, muchas de las empresas optaron por la primera alternativa. De ahí que se haya hecho necesario tomar datos de las dos opciones. 


\section{Tabla 2}

\section{Tamaño de los grupos empresariales atendiendo al número de empleados}

\begin{tabular}{lcc}
\hline Agrupamientos de grupos empresariales & $\mathrm{N}^{\circ}$ de empleados & $\mathrm{N}^{\circ}$ de grupos empresariales \\
\hline Nivel 1 Tamaño pequeño & $0-4.267$ & 45 \\
Nivel 2 Tamaño mediano & $4.267-28.686$ & 46 \\
Nivel 3 Tamaño grande & $28.686-391.544$ & 45 \\
\hline
\end{tabular}

Fuente: elaboración propia, a partir de SPSS v. 17.

dimensión, pero como resulta conocido los requisitos exigidos por el NASDAQ para la admisión a cotización son inferiores a los requeridos por NYSE, y así pueden cotizar empresas en dicho mercado de menor tamaño.

En concreto, la compañía de navegación griega Tsakos Energy Navigation, por cierto, cotizada en NYSE, no contó con ningún empleado asalariado para ninguno de los años del período 19992004, mientras que el grupo empresarial de más empleados, fue el grupo alemán denominado en su día DaimlerChrysler, que en el año 1999 contaba con 446.938.

Por todo ello, se está ante un conjunto de grupos empresariales de reducidas dimensiones relativas, junto a otros que se pueden calificar de muy gran dimensión, que por otra parte enriquecerán los contrastes empíricos asociados al tamaño al contar en la muestra con grupos empresariales de todas las dimensiones.

Antes de entrar a justificar las herramientas con las que se practicarán los contrastes en esta investigación, se hace necesario explicar las variables sobre las que se practicarán los mismos. Tal como se ha señalado, a cada grupo empresarial se le toman el resultado global y el resultado neto conforme a US GAAP para cada uno de los años del período 19992004.

Sin embargo, se necesita construir una nueva variable para practicar los contrastes, a partir de las dos anteriores, en concreto, se trata de la discrepancia en términos relativos entre el resultado global-comprehensive income- $(\mathrm{Cl})$ y el resultado neto - net income- $(\mathrm{NI})$, medida a través de la relación $[(\mathrm{Cl}-\mathrm{NI}) / / \mathrm{NI} 100]$, en adelante $\mathrm{CINI}$, particularizada para cada uno de los años del referido período.

Se han tomado valores relativos en lugar de absolutos para con ello definir la variable CINI en cada uno de los años de tal modo que ésta refleje la auténtica discrepancia para cada empresa entre ambos tipos de resultado.

Por otra parte, tanto las variables $\mathrm{Cl}$ y NI, pueden tomar valores positivos $\mathrm{y}$ negativos, por lo que se ha hecho necesario trabajar con valores absolutos en el denominador, para así poder determinar correctamente en todos los casos la discrepancia relativa real entre ambos tipos de resultado.

Asimismo, con el objeto de apoyar y justificar los resultados de los contrastes que practiquemos, también se han tomado los datos de cada uno de los ítems novedosos que incorpora el resultado 
global en los resultados de las empresas, para proceder después a su relativización con respecto al resultado neto.

En concreto, como ya se hizo referencia a algunos de ellos, se trata de las diferencias de conversión de moneda extranjera (ME), de las inversiones financieras disponibles para la venta (IF), de los derivados y operaciones de cobertura (DE) y del fondo de pensiones (FP), que se relativizan con respecto al resultado neto a través de las siguientes expresiones: $\mathrm{MENI}=[\mathrm{ME} / \mathrm{NI} \cdot 100] ; \mathrm{IFNI}=\mathrm{IF} / \mathrm{NI} \mid$. $100] ; \mathrm{DENI}=[\mathrm{DE} / \mathrm{NI} \cdot \cdot 100]$ y $\mathrm{FPNI}=[\mathrm{FP}$ $\mid \mathrm{NI} \cdot 100]$, respectivamente, teniendo en cuenta sus valores para cada uno de los años del período 1999-2004.

Asimismo, aunque los 136 grupos empresariales incluidos en la muestra revelaron conforme al SFAS 130 estos ítems perfectamente delimitados, un reducido número de ellos incluyeron la partida "otros", que no se incluyen en el análisis por corresponder a partidas de distinta naturaleza y representar tan solo alrededor de un uno por ciento del resultado neto para el conjunto del período 19992004, que evidentemente supondrán desajustes mínimos dado que la variable CINI integra a MENI, IFNI, DENI, FPNI, y a "otros", pero esta última partida no influye en el análisis por no tener apenas importancia relativa.
Por otra parte, para llevar a cabo el contraste estadístico, dado que se está ante un factor inter-sujetos en el sentido de que no todos los sujetos, en este caso los grupos empresariales, pasan por los mismos tratamientos, y evidentemente cada uno de ellos queda asociado a uno de los tres niveles por tamaño, podría resultar adecuado plantear un modelo ANOVA de una vía para probar la existencia o no de diferencias estadísticamente significativas entre múltiples grupos.

Asimismo, como se toman valores a cada grupo empresarial de forma repetida a lo largo de los años del período 1999-2004, también podría resultar adecuado plantear un modelo ANOVA de medidas repetidas, empleando como factor intra-sujetos el año y como factor inter-sujetos el tamaño.

Sin embargo, al practicar la prueba de Kolmogorov-Smirnov para una muestra, se rechaza la hipótesis nula de normalidad de las variables CINI para cada uno de los años del período 1999-2004. Asimismo, en la Tabla 3 queda también patente el claro alejamiento de la normalidad de los datos al estar ante un elevado número de valores extremos y atípicos -outliers-, que se han identificado a partir del análisis de los correspondientes diagramas de caja o boxplots ${ }^{2}$.

2 Por una parte, se han marcado los valores extremos con un asterisco $\left({ }^{*}\right)$, que concretamente, tal como se refiere en SPSS v.17, son casos con valores superiores a 3 longitudes de caja desde el borde superior o inferior de la caja, teniendo en cuenta que la longitud de la misma es la amplitud intercuartil; y de otra, se han señalado con un círculo $(O)$ los valores atípicos o outliers que corresponden a casos con valores entre 1,5 y 3 longitudes de caja desde el borde superior o inferior de la caja, siendo también la longitud de la caja la amplitud intercuartil. 


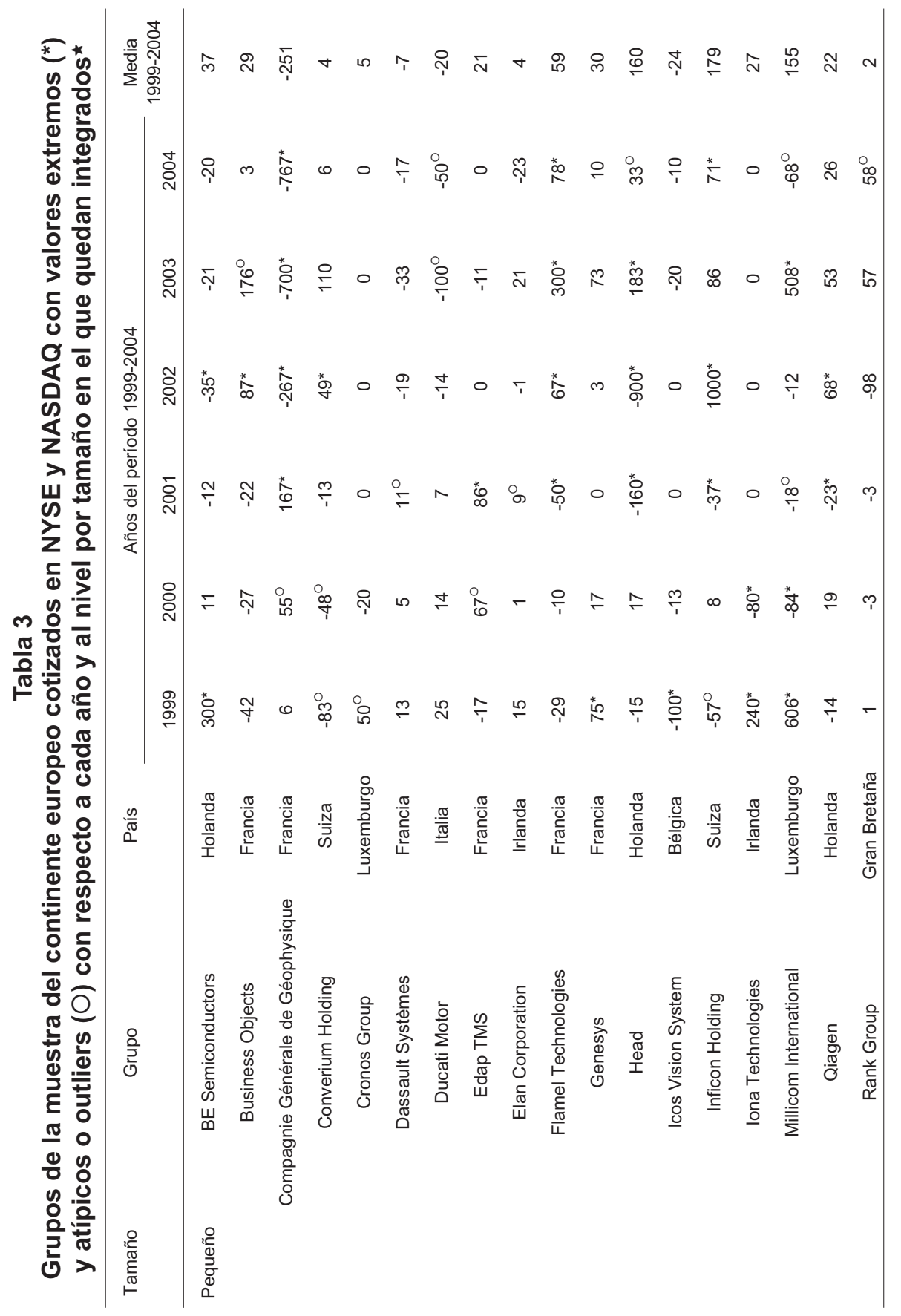




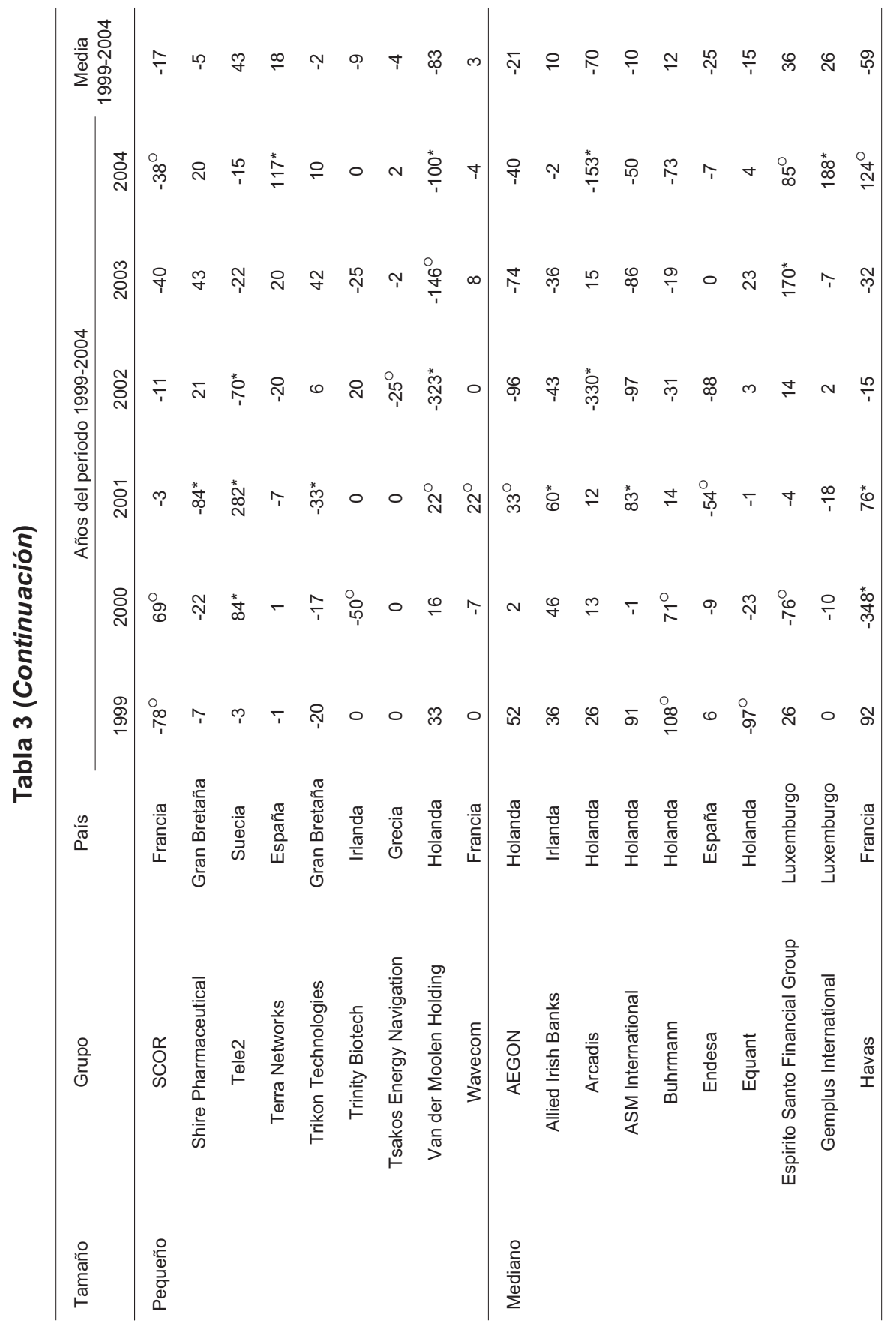




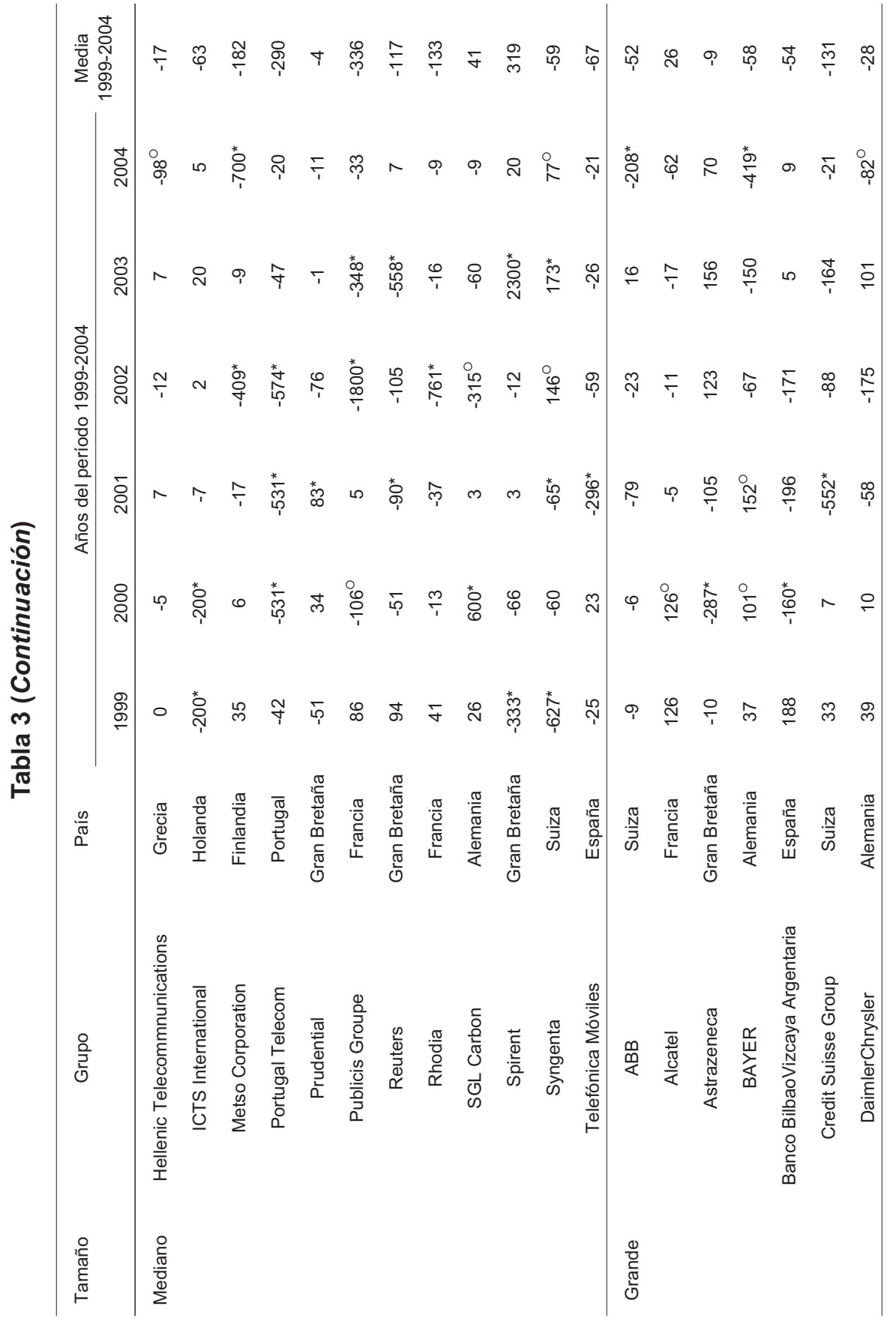




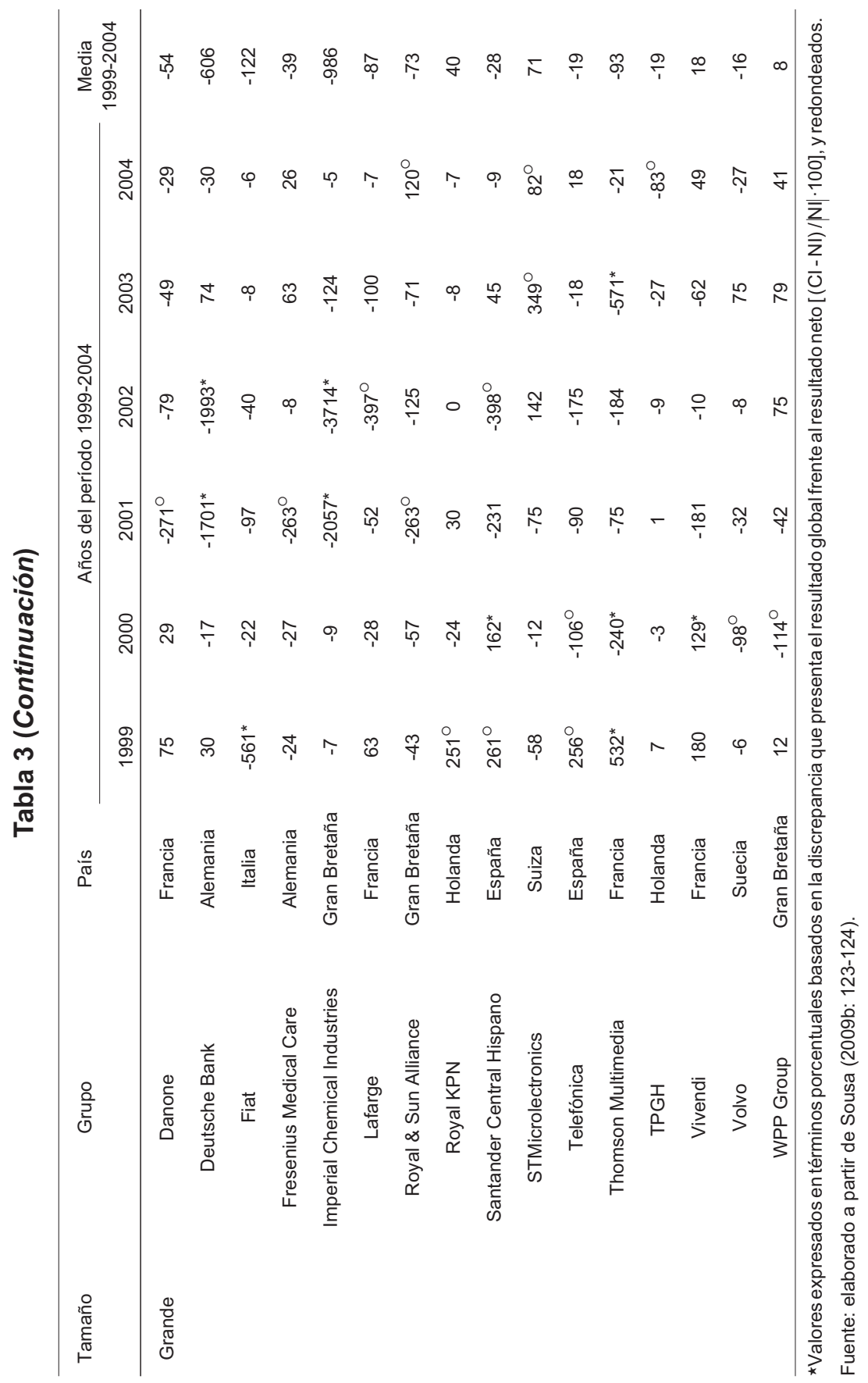


En efecto, se está ante un considerable número de grupos, más del sesenta y dos por ciento de la muestra, que registraron valores extremos y/o atípicos (outliers) en algunos años en relación al agrupamiento por tamaño, que responden al impacto relativo en los mismos del resultado global frente al resultado neto, en algunos casos muy acusado y en otros puede calificarse de espectacular.

Por lo tanto, como los datos están muy alejados de una distribución gaussiana, que es una asunción para poder utilizar modelos ANOVA, se ha de aplicar el test alternativo en herramientas no paramétricas, la prueba de Kruskal-Wallis, conocida también como el análisis de la varianza de una vía por rangos de Kruskal-Wallis (Siegel y Castellán, 1988). Este test permite constatar si un factor, en este caso el factor dimensión empresarial, que subdivide a la población de origen, afecta significativamente a su valor central.

Pero el test de Kruskal-Wallis asume que las variables objeto de contraste se distribuyan de forma similar entre los diferentes grupos, en este caso, entre los diferentes niveles por tamaño. De acuerdo con el estudio de los correspondientes diagramas de caja, que queda sintetizado en la Tabla 3, se está lejos del cumplimiento de esta asunción dado que muchos valores extremos y atípicos se distribuyen de una forma bastante irregular entre los distintos niveles por tamaño.

Por lo tanto, se hace necesario validar los resultados que se obtengan en la prueba de Kruskal-Wallis con el Test de la Mediana, que no requiere la asunción que se ha referido con anterioridad; pero su debilidad es que no está diseñada para tener en cuenta la distancia desde la mediana, lo que sí tiene en cuenta la prueba de Kruskal-Wallis.

En definitiva, de las dos alternativas con las que se cuenta en SPSS v.17 para practicar los contrastes, una exige la distribución de las variables con una forma similar entre los grupos, y la otra es más flexible al prescindir de este requisito pero presenta el punto débil que se ha indicado, con lo que para robustecer las conclusiones que se extraigan se hace necesario apoyarse en los resultados de ambas.

Si estas dos pruebas a priori muestran diferencias estadísticamente significativas entre los distintos niveles por tamaño con respecto al impacto relativo del resultado global frente al resultado neto, trabajando con un nivel de confianza del $95 \%$, por lo que la significatividad vendrá determinada por $p<0,05$; se han de practicar los correspondientes tests post hoc para con ello determinar entre qué pares de sectores y años concretos se producen las diferencias significativas.

Para ello, resulta adecuado practicar la prueba de la $U$ de Mann-Whitney, que permite contrastar la hipótesis nula de que dos muestras independientes proceden de la misma población. Sin embargo, este test asume que las variables objeto de contraste tengan una distribución similar entre los dos grupos que se comparan, en este caso, entre los diferentes pares por niveles de tamaño. Pero los datos están lejos de cumplir este requisito por las mismas razones que se han dado para los test a priori. 
En consecuencia, se va a utilizar el test de Kolmogorov-Smirnov para dos muestras para validar la asunción de distribución similar, que contrariamente a la prueba de la $U$ de Mann-Whitney no requiere esta condición, lo que confiere más flexibilidad pero al mismo tiempo esta última prueba es muy sensible tanto a diferencias de localización como de escala.

En definitiva, de las dos alternativas con las que se cuenta en SPSS v.17 para practicar los contrastes, una exige la distribución de las variables con una forma similar entre los grupos, y la otra es más flexible al prescindir de este requisito pero presenta el punto débil que se ha indicado, con lo que para robustecer las conclusiones que se extraigan se hace necesario apoyarse en los resultados de ambas.

\section{Resultado global en grupos empresariales según dimensión: evidencia empírica}

Antes de entrar en el análisis de los resultados de la investigación, se analizan los estadísticos descriptivos de las variables que han servido de base para realizar los contrastes. De este modo, en la Tabla 4 queda recogido el comportamiento del resultado global y del resultado neto para los 136 grupos de la muestra.

Cabe destacar el acusado impacto negativo del resultado global frente al resultado neto para el año 2001 , y en particular, para el año 2002, que hace empeorar los resultados de los grupos empresariales de forma que se puede calificar de espectacular, como se verá después, motivado por las fuertes caídas bursáti-

\section{Tabla 4}

\section{Estadísticos descriptivos del resultado neto (NI) y resultado global (Cl) los años del período 1999-2004 para los grupos europeos cotizados en NYSE y NASDAQ*}

\begin{tabular}{lrrrrrrrrrr}
\hline & N & \multicolumn{1}{c}{ Media } & Mediana & D.E. & Mínimo & Máximo & Rango & A.I. & Asimetría & Curtosis \\
\hline NI1999 & 136 & 508,10 & 112,00 & $1.034,18$ & -3.523 & 5.746 & 9.269 & 630 & 1,83 & 7,97 \\
Cl1999 & 136 & 647,80 & 114,00 & $1.315,31$ & -3.894 & 7.988 & 11.882 & 868 & 2,24 & 9,49 \\
NI2000 & 136 & 970,60 & 160,00 & $2.148,85$ & -1.236 & 13.513 & 14.749 & 869 & 3,44 & 13,66 \\
Cl2000 & 136 & 908,86 & 101,00 & $2.005,69$ & -1.738 & 11.244 & 12.982 & 840 & 2,72 & 8,22 \\
NI2001 & 136 & $-124,89$ & 29,00 & $2.531,40$ & -19.278 & 6.317 & 25.595 & 475 & $-4,32$ & 28,57 \\
Cl2001 & 136 & $-371,61$ & 11,00 & $2.748,23$ & -18.157 & 6.795 & 24.952 & 526 & $-3,87$ & 21,19 \\
NI2002 & 136 & $-545,11$ & 22,50 & $5.253,55$ & -43.857 & 8.007 & 51.864 & 575 & $-6,23$ & 45,50 \\
Cl2002 & 136 & $-1.182,33$ & $-6,00$ & $5.907,31$ & -48.051 & 10.054 & 58.105 & 718 & $-5,87$ & 40,99 \\
NI2003 & 136 & 549,83 & 118,50 & $1.587,46$ & -3.670 & 10.406 & 14.076 & 559 & 2,80 & 13,14 \\
Cl2003 & 136 & 500,27 & 85,00 & $1.883,21$ & -3.946 & 15.905 & 19.851 & 563 & 4,36 & 33,35 \\
NI2004 & 136 & 827,80 & 192,00 & $1.708,16$ & -2.100 & 12.547 & 14.647 & 967 & 3,50 & 17,68 \\
Cl2004 & 136 & 783,49 & 141,50 & $1.736,66$ & $-2 . .223$ & 12.754 & 14.977 & 815 & 3,38 & 17,21 \\
\hline
\end{tabular}

*Valores según US GAAP, expresados en millones de euros para cada uno de los años.

D.E. Desviación estándar. A.I. Amplitud intercuartil. 
les, las diferencias de conversión de moneda extranjera negativas y los ajustes también negativos de la cobertura mínima del fondo de pensiones de los empleados.

Además, se cuenta con valores máximos y mínimos muy extremos, así como desviaciones estándares y asimetrías y curtosis muy elevadas, que indican la no normalidad de los datos, ya estudiada con anterioridad, lo que también supone que para determinados grupos empresariales el resultado global ha impactado frente al resultado neto a lo largo del período considerado de forma acusada.

Por otra parte, en la Tabla 5 y en el Gráfico 1, se ha querido recoger el impacto relativo del resultado global frente al re-

Tabla 5

\section{Estadísticos descriptivos asociados a la variable CINI los años del período 1999-2004 para los grupos europeos cotizados en NYSE y NASDAQ*}

\begin{tabular}{ccrrrrrrrrr}
\hline CINI & N & Media & Mediana & \multicolumn{1}{c}{ D.E. } & \multicolumn{1}{c}{ Mínimo } & Máximo & Rango & A.I. & Asimetria & Curtosis \\
\hline CINI1999 & 136 & 15,41 & 0,00 & 125,43 & $-626,56$ & 605,88 & $1.232,44$ & 47,97 & $-0,30$ & 12,97 \\
CINI2000 & 136 & $-10,96$ & 0,00 & 92,63 & $-530,00$ & 600,00 & $1.130,00$ & 28,87 & 0,16 & 21,94 \\
CINI2001 & 136 & $-54,87$ & $-2,94$ & 244,08 & $-2.057,14$ & 281,71 & $2.338,85$ & 39,03 & $-6,56$ & 48,32 \\
CINI2002 & 136 & $-93,56$ & $-18,54$ & 421,10 & $-3.714,29$ & $1.000,00$ & $4.714,29$ & 69,30 & $-5,78$ & 45,22 \\
CINI2003 & 136 & 10,09 & 0,00 & 236,74 & $-700,00$ & $2.300,00$ & $3.000,00$ & 66,64 & 6,46 & 66,17 \\
CINI2004 & 136 & $-18,26$ & $-1,67$ & 105,25 & $-766,67$ & 187,50 & 954,17 & 28,23 & $-5,10$ & 32,31 \\
\hline
\end{tabular}

*Valores de [ $(\mathrm{Cl}-\mathrm{NI}) / \mid \mathrm{NI} \cdot$ - 100] según US GAAP, expresados evidentemente en términos relativos.

D.E. Desviación estándar. A.I. Amplitud intercuartil.

Fuente: elaboración propia, a partir de SPSS v.17.

\section{Gráfico 1}

Impacto del resultado global frente al resultado neto los años del período 1999-2004 para los grupos europeos cotizados en NYSE y NASDAQ

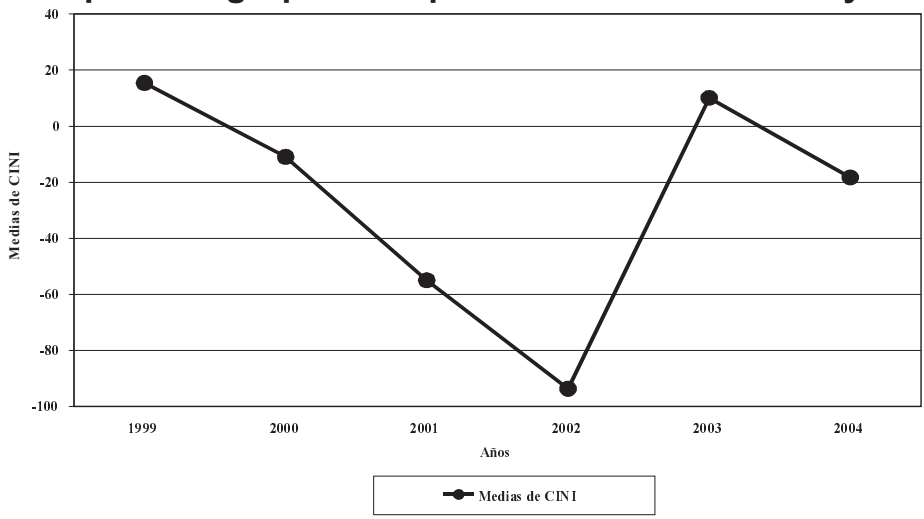

Valores de $[(\mathrm{Cl}-\mathrm{NI}) / \mathrm{NI} \mid \cdot 100]$ según US GAAP, expresados evidentemente en términos relativos Fuente: elaboración propia, a partir de Sousa (2009b: 120). 
sultado neto capturado por la variable CINI según quedó definida.

En coherencia con los datos anteriores, se observa un acusado impacto relativo del resultado global frente al resultado neto, en particular los años 2001 y 2002, que supone un empeoramiento de los resultados de los grupos empresariales de forma espectacular, cerca del $55 \%$ y del $94 \%$ respectivamente.

Asimismo, para todo el período de estudio y los 136 grupos empresariales, se está ante el hecho tan destacado que el resultado global ha empeorado los resultados de los grupos empresariales, frente el más tradicional resultado neto, en más de un $25 \%$.

Tal como se recoge en la Tabla 6 , dicho empeoramiento se debe fundamentalmente a las fuertes caídas bursátiles de principios de los dos mil con fuerte impacto negativo en la partida de las inversiones financieras clasificadas como disponibles para la venta, los tipos de cambio desfavorables al convertir activos y pasivos de las filiales extranjeras en el proceso de consolidación, sin olvidar el peso de los ajustes negativos de la cobertura mínima del fondo de pensiones de los empleados.

\section{Tabla 6}

\section{Estadísticos descriptivos correspondientes a los elementos que incorpora el resultado global en los resultados empresariales relativizados con respecto al resultado neto ${ }^{* 3}$}

\begin{tabular}{|c|c|c|c|c|c|}
\hline Años & $\mathrm{N}^{\circ}$ & $\begin{array}{l}\text { Diferencias de } \\
\text { conversión en } \\
\text { moneda } \\
\text { extranjera } \\
\mathrm{MENI}= \\
{[\mathrm{ME} / \mathrm{NII} \cdot 100]}\end{array}$ & $\begin{array}{c}\text { Beneficios y pérdidas } \\
\text { no realizados en } \\
\text { inversiones financieras } \\
\text { disponibles para la } \\
\text { venta } \\
\text { IFNI = [ IF / } / \mathrm{NI} \mid \cdot 100]\end{array}$ & $\begin{array}{c}\text { Ajuste de cobertura } \\
\text { mínima del fondo de } \\
\text { pensiones de los } \\
\text { empleados } \\
\text { FPNI = } \\
{[D E /|N| \cdot 100]}\end{array}$ & $\begin{array}{c}\text { Beneficios y pérdidas } \\
\text { de Derivados y } \\
\text { Coberturas de Flujos } \\
\text { de Caja } \\
\text { DENI = } \\
{[D E / N I \mid \cdot 100]}\end{array}$ \\
\hline 1999 & 136 & $-3,90$ & 19,77 & 0,45 & $-1,49$ \\
\hline 2000 & 136 & $-4,00$ & $-6,36$ & $-0,50$ & 0,38 \\
\hline 2001 & 136 & $-9,15$ & $-31,25$ & $-16,34$ & $-1,58$ \\
\hline 2002 & 136 & $-39,27$ & $-23,48$ & $-37,99$ & 2,12 \\
\hline 2003 & 136 & $-19,22$ & 13,68 & 11,48 & 4,01 \\
\hline 2004 & 136 & $-13,47$ & $-0,90$ & $-3,54$ & 0,43 \\
\hline $\begin{array}{c}\text { Media } \\
1999-2004\end{array}$ & 136 & $-14,84$ & $-4,76$ & $-7,74$ & 0,65 \\
\hline
\end{tabular}

*Valores según US GAAP, expresados en términos porcentuales.

Fuente: elaboración propia, a partir de Sousa (2009b: 120).

3 La suma de las medias de estos cuatro ítems es de $-26,69$, que no coincide con la media global para todo el período de estudio que es de -25,36. La diferencia de 1,33, corresponde con el ítem "otros", que no se ha considerado en la investigación por tener escasa importancia relativa. 
Una vez que ha quedado analizado el comportamiento del resultado global frente al resultado neto de una forma general para todos los grupos empresaria- les, se entra ahora a analizar el efecto incorporando la variable tamaño, tal como queda recogido en la Tabla 7 y en los Gráficos 2,3 y 4 .

\section{Tabla 7}

\section{Medias del factor tamaño en el período 1999-2004 sobre la discrepancia relativa del resultado global frente al resultado neto medida a través de $\mathrm{CINI}{ }^{4}$}

\begin{tabular}{lrrrrrrr}
\hline \multicolumn{1}{c}{ Tamaño } & CINI1999 & CINI2000 & CINI2001 & CINI2002 & CINI2003 & CINI2004 & $\begin{array}{c}\text { Media } \\
1999-2004\end{array}$ \\
\hline Pequeño & 17,62 & 0,35 & 3,18 & 31,91 & 13,01 & $-15,59$ & 8,41 \\
Mediano & $-12,09$ & $-18,24$ & $-19,32$ & $-121,31$ & 36,01 & $-24,40$ & $-26,56$ \\
Grande & 41,30 & $-14,84$ & $-149,26$ & $-190,66$ & $-19,33$ & $-14,64$ & $-57,91$ \\
Media por años & 15,41 & $-10,96$ & $-54,87$ & $-93,56$ & 10,09 & $-18,26$ & $-25,36$ \\
\hline
\end{tabular}

*Valores de [ ( $\mathrm{Cl}-\mathrm{NI}) / \mid \mathrm{NI} \cdot$ - 100] según US GAAP, expresados evidentemente en términos relativos. Fuente: elaboración propia, a partir de Sousa (2009b: 122).

\section{Gráfico 2}

Perfil de las medias de la discrepancia relativa del resultado global frente al resultado neto en el período 1999-2004 para el factor tamaño

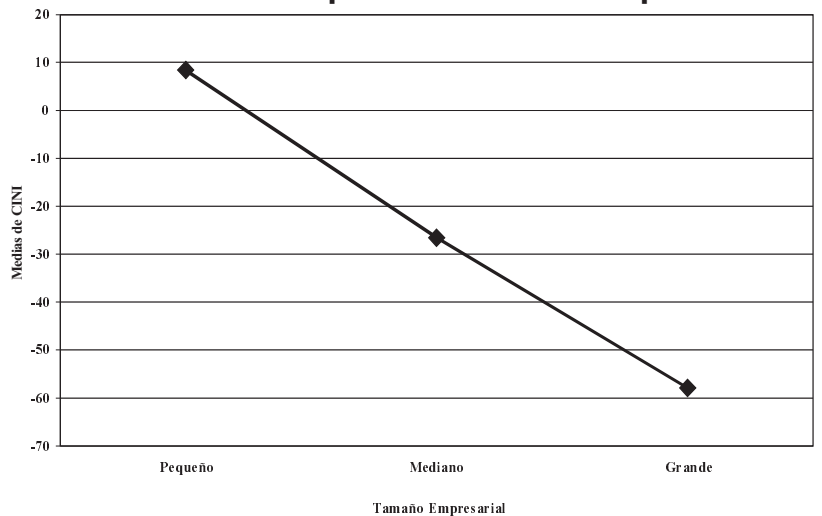

Valores de $[(\mathrm{Cl}-\mathrm{NI}) / \mathrm{NI} \cdot$ - 100] según US GAAP, expresados evidentemente en términos relativos Fuente: elaboración propia, a partir de SPSS v.17.

4 Se quiere advertir que al no contar con el mismo número de grupos empresariales en cada uno de los tres niveles por tamaño (45 pequeños, 46 medianos y 45 grandes), para determinar las medias tanto por años como por cada nivel por tamaño no se utilizan evidentemente las medias aritméticas simples, sino las ponderadas. 


\section{Gráfico 3}

Perfil de las medias de la discrepancia relativa del resultado global frente al resultado neto a lo largo de los años del período 1999-2004 para el factor tamaño

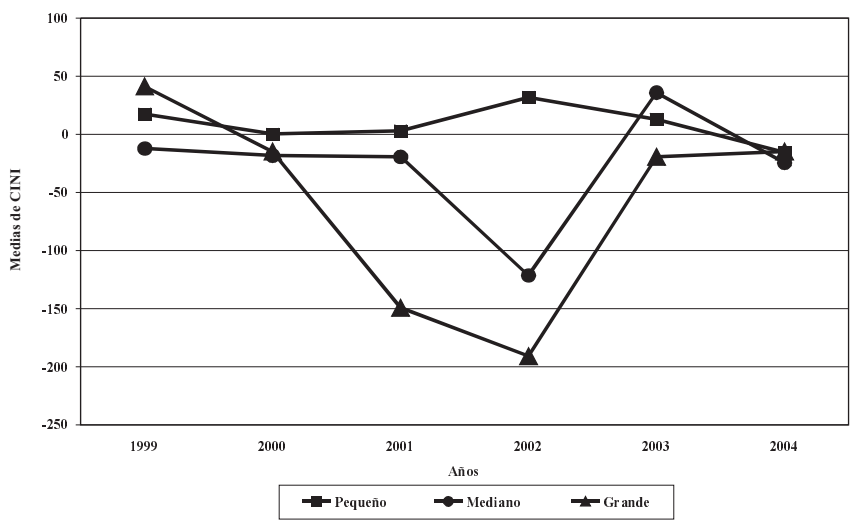

Valores de $[(\mathrm{Cl}-\mathrm{NI}) / \mathrm{NI} \cdot 100]$ según US GAAP, expresados evidentemente en términos relativos Fuente: elaboración propia, a partir de Sousa (200b: 122).

\section{Gráfico 4}

Perfil de las medias de la discrepancia relativa del resultado global frente al resultado neto a lo largo de los años del período 1999-2004 para el factor tamaño

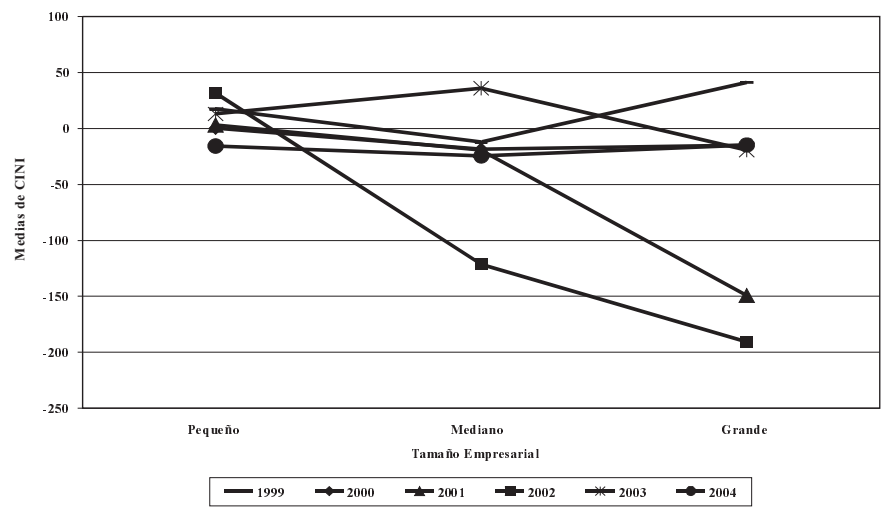

Valores de $[(\mathrm{Cl}-\mathrm{NI}) / \mathrm{NI} \cdot$ 100] según US GAAP, expresados evidentemente en términos relativos Fuente: elaboración propia, a partir de SPSS v.17.

Se puede observar que por término medio para los grupos empresariales de menor dimensión el resultado global supera relativamente al resultado neto, mientras que en sentido contrario, para los de mediana y gran dimensión el resultado global es claramente inferior al resultado neto, con un acusado impacto nega- 
tivo para los medianos, y en particular, paras los grandes.

Asimismo, el impacto es bastante estable a lo largo de los años estudiados para los grupos empresariales pequeños, mientras que para los medianos y de gran dimensión es mucho más variable, hasta el punto de suponer una volatilidad bastante acusada en la cifra de resultados revelada a través del tiempo.

Por otra parte, se quiere profundizar más en las causas que puedan justificar las diferencias por tamaño que se están detectando, para lo que se va a estudiar ahora el comportamiento de cada uno de los ítems que incorpora el resulta- do global en resultados frente al resultado neto, particularizados para la dimensión de los grupos empresariales, con los resultados que se recogen en las Tablas 8 , 9, 10 y 11 .

En congruencia con los estadísticos descriptivos que se vienen comentando, resulta evidente el menor impacto de los ítems novedosos que incorpora el resultado global en resultados sobre el resultado neto en los grupos empresariales pequeños frente a los medianos, y en especial con respecto a los grandes; y también el impacto globalmente menor de los medianos frente a los de gran dimensión.

\section{Tabla 8}

Impacto por tamaño de las medias para el período 1999-2004 de la discrepancia relativa de la partida diferencias de conversión en moneda extranjera frente al resultado neto*

\begin{tabular}{lrrrrrrr}
\hline Tamaño & MENI1999 & MENI2000 & MENI2001 & MENI2002 & MENI2003 & MENI2004 & $\begin{array}{c}\text { Media } \\
1999-2004\end{array}$ \\
\hline Pequeño & 1,00 & 2,44 & 2,05 & 34,37 & $-9,22$ & $-7,47$ & 3,86 \\
Mediano & $-18,04$ & $-10,34$ & $-8,03$ & $-87,75$ & $-11,36$ & $-27,20$ & $-27,12$ \\
Grande & 5,65 & $-3,96$ & $-21,48$ & $-63,36$ & $-37,25$ & $-5,44$ & $-20,97$ \\
Total & $-3,90$ & $-4,00$ & $-9,15$ & $-39,27$ & $-19,22$ & $-13,47$ & $-14,84$ \\
\hline
\end{tabular}

*Valores de ME $=[(\mathrm{ME}) / \mathrm{NI} \cdot 100]$ según US GAAP, expresados evidentemente en términos relativos. Fuente: elaboración propia, a partir de SPSS v.17.

\section{Tabla 9}

Impacto por tamaño de las medias para el período 1999-2004 de la discrepancia relativa de la partida inversiones financieras disponibles para la venta frente al resultado neto*

\begin{tabular}{lrrrrrrr}
\hline Tamaño & IFNI1999 & IFNI2000 & IFNI2001 & IFNI2002 & IFNI2003 & IFNI2004 & $\begin{array}{c}\text { Media } \\
1999-2004\end{array}$ \\
\hline Pequeño & 16,62 & $-2,45$ & 1,43 & $-1,97$ & 20,12 & $-7,33$ & 4,40 \\
Mediano & 8,35 & $-6,98$ & $-4,46$ & $-10,54$ & 9,69 & 3,80 & $-0,02$ \\
Grande & 34,61 & $-9,65$ & $-91,30$ & $-58,21$ & 11,30 & 0,74 & $-18,75$ \\
Total & 19,77 & $-6,36$ & $-31,25$ & $-23,48$ & 13,68 & $-0,90$ & $-4,76$ \\
\hline
\end{tabular}

*Valores de IF $=[(\mathrm{IF}) / / \mathrm{NI} \cdot 100]$ según US GAAP, expresados evidentemente en términos relativos.

Fuente: elaboración propia, a partir de SPSS v.17. 


\section{Tabla 10}

Impacto por tamaño de las medias para el período 1999-2004 de la discrepancia relativa de la partida derivados y operaciones de cobertura frente al resultado neto*

\begin{tabular}{lccccccc}
\hline Tamaño & DENI1999 & DENI2000 & DENI2001 & DENI2002 & DENI2003 & DENI2004 & $\begin{array}{c}\text { Media } \\
1999-2004\end{array}$ \\
\hline Pequeño & 0,00 & 0,24 & 0,27 & 0,16 & 1,20 & $-0,78$ & 0,18 \\
Mediano & $-4,31$ & 0,97 & $-0,93$ & 4,50 & 3,60 & 3,39 & 1,20 \\
Grande & $-1,10$ & $-0,09$ & $-4,08$ & 1,66 & 7,25 & $-1,38$ & 0,38 \\
Total & $-1,49$ & 0,38 & $-1,58$ & 2,12 & 4,01 & 0,43 & 0,65 \\
\hline
\end{tabular}

*Valores de $\mathrm{DE}=[(\mathrm{DE}) / \mathrm{NI} \cdot \cdot 100]$ según US GAAP, expresados evidentemente en términos relativos.

Fuente: elaboración propia, a partir de SPSS v.17.

Tabla 11

Impacto por tamaño de las medias para el período 1999-2004 de la discrepancia relativa de la partida fondo de pensiones frente al resultado neto*

\begin{tabular}{lccccccc}
\hline Tamaño & FPNI1999 & FPNI2000 & FPNI2001 & FPNI2002 & FPNI2003 & FPNI2004 & $\begin{array}{c}\text { Media } \\
1999-2004\end{array}$ \\
\hline Pequeño & 0,00 & 0,12 & $-0,56$ & $-0,64$ & 0,91 & $-0,01$ & $-0,03$ \\
Mediano & 0,13 & $-0,63$ & $-3,10$ & $-27,17$ & 33,76 & $-2,00$ & 0,16 \\
Grande & 1,23 & $-0,99$ & $-45,67$ & $-86,41$ & $-0,74$ & $-8,65$ & $-23,54$ \\
Total & 0,45 & $-0,50$ & $-16,34$ & $-37,99$ & 11,48 & $-3,54$ & $-7,74$ \\
\hline
\end{tabular}

*Valores de FP $=[(\mathrm{FP}) / / \mathrm{NI} \mid \cdot 100]$ según US GAAP, expresados evidentemente en términos relativos.

Fuente: elaboración propia, a partir de SPSS v.17.

Asimismo, cabe destacar los notables efectos negativos de la partida de diferencias de conversión en moneda extranjera en los grupos empresariales medianos y grandes, y también un impacto negativo considerable en estos últimas de las partidas de inversiones financieras disponibles para la venta y fondo de pensiones; sin embargo, las consecuencias son prácticamente irrelevantes teniendo en cuenta las medias de la partida de derivados y cobertura en los tres niveles por tamaño considerados.

Una vez que se han analizado los estadísticos descriptivos del impacto del resultado global frente al resultado neto, en particular, incorporando la variable tamaño, se procede ahora al estudio de los contrastes estadísticos asociados al objetivo que se ha propuesto en esta investigación.

Como se está ante un factor intersujetos, tal como quedó indicado, se practica en principio la prueba de Kruskal-Wallis para determinar si se presentan diferencias estadísticamente significativas entre los distintos niveles por tamaño y cómo se comportan a lo largo de los años, con respecto al impacto relativo del resultado global frente al resulta- 
do neto, cuyos resultados aparecen recogidos en las Tablas 12 y 13.

Como se puede observar en la Tabla 12, en el año 1999 los grupos empresariales de gran dimensión cuentan con la mayor suma de rangos, y con ello unas mayores discrepancias positivas entre el resultado global frente al resultado neto, seguidos por los de tamaño mediano, y ya a una mayor distancia por los pequeños.

Sin embargo, para el resto de años la tendencia es la contraria, en especial para los años 2001 y 2002, en los que se registran las máximas diferencias entre los rangos promedio, y con ello, los grupos empresariales pequeños, en conjunto, presentan para esos años mayores discrepancias positivas del resultado global frente al resultado neto que los grupos empresariales grandes y medianos.
Estas diferencias notables de rangos entre los distintos niveles por tamaño los referidos años, tal como se recoge en la Tabla 13, conducen a $p<0,05$; y por lo tanto llevan a resultados significativos para los años 1999, 2001 y 2002.

Asimismo, como las variables no se distribuyen de forma similar entre los tres niveles por tamaño, tal como quedó justificado, se ha considerado adecuado practicar el Test de la Mediana para complementar y dar refuerzo a las anteriores revelaciones, con los resultados que se recogen en las Tablas 14 y 15.

Así, en la Tabla 14 se observa un fenómeno muy similar al que describió la prueba de Kruskal-Wallis, en el sentido de que en el año 1999 los grupos empresariales de gran dimensión cuentan con el mayor número de casos que exceden de la mediana, y con ello registran unas

Tabla 12

Rangos promedio de la Prueba de Kruskal-Wallis para el factor tamaño

\begin{tabular}{lccccccc}
\hline \multirow{2}{*}{ Tamaño } & \multirow{2}{*}{$N^{\circ}$} & \multicolumn{5}{c}{ Rangos Promedio } \\
\cline { 3 - 7 } & & CINI1999 & CINI2000 & CINI2001 & CINI2002 & CINI2003 & CINI2004 \\
\hline Pequeño & 45 & 57,76 & 74,26 & 81,46 & 94,99 & 76,14 & 77,40 \\
Mediano & 46 & 67,63 & 63,58 & 75,10 & 57,21 & 68,08 & 61,67 \\
Grande & 45 & 80,13 & 67,78 & 48,80 & 53,56 & 61,29 & 66,58 \\
Total & 136 & & & & & & \\
\hline
\end{tabular}

Fuente: elaboración propia, a partir de SPSS v.17.

Tabla 13

Estadísticos de contraste de la Prueba de Kruskal-Wallis para el factor tamaño

\begin{tabular}{lcccccc}
\hline & CINI1999 & CINI2000 & CINI2001 & CINI2002 & CINI2003 & CINI2004 \\
\hline Chi-cuadrado & 7,305 & 1,695 & 17,442 & 30,629 & 3,210 & 3,788 \\
gl & 2 & 2 & 2 & 2 & 2 & 2 \\
Sig. asintót. & 0,026 & 0,429 & 0,000 & 0,000 & 0,201 & 0,150 \\
\hline
\end{tabular}

Fuente: elaboración propia, a partir de SPSS v.17. 
Tabla 14

Frecuencias de la Prueba de la Mediana para el factor tamaño

\begin{tabular}{llccc}
\hline & & \multicolumn{3}{c}{ Tamaño } \\
\cline { 3 - 5 } & & Pequeño & Mediano & Grande \\
\hline \multirow{2}{*}{ CINI1999 } & $>$ Mediana & 12 & 25 & 30 \\
CINI2000 & $\leq$ Mediana & 33 & 21 & 15 \\
& $>$ Mediana & 18 & 18 & 23 \\
CINI2001 & $\leq$ Mediana & 27 & 28 & 22 \\
CINI2002 & $>$ Mediana & 31 & 24 & 13 \\
& $\leq$ Mediana & 14 & 22 & 32 \\
CINI2003 & $>$ Mediana & 37 & 15 & 16 \\
& $\leq$ Mediana & 8 & 31 & 29 \\
CINI2004 & $>$ Mediana & 18 & 20 & 27 \\
& $\leq$ Mediana & 27 & 26 & 19 \\
& $>$ Mediana & 32 & 17 & 26 \\
\hline
\end{tabular}

Fuente: elaboración propia, a partir de SPSS v.17

Tabla 15

Estadísticos de contraste de la Prueba de la Mediana para el factor tamaño

\begin{tabular}{lcccccc}
\hline & CINI1999 & CINI2000 & CINI2001 & CINI2002 & CINI2003 & CINI2004 \\
\hline $\mathrm{N}$ & 136 & 136 & 136 & 136 & 136 & 136 \\
Mediana & 0,0000 & 0,0000 & $-2,9376$ & $-18,5443$ & 0,0000 & $-1,6748$ \\
Chi-cuadrado & $15,122(\mathrm{a})$ & $1,643(\mathrm{~b})$ & $14,531(\mathrm{c})$ & $28,010(\mathrm{c})$ & $0,152(\mathrm{~d})$ & $12,242(\mathrm{c})$ \\
gl & 2 & 2 & 2 & 2 & 2 & 2 \\
Sig. asintót. & 0,001 & 0,440 & 0,001 & 0,000 & 0,927 & 0,002 \\
\hline
\end{tabular}

a 0 casillas $(0 \%)$ tienen frecuencias esperadas menores que 5 . La frecuencia de casilla esperada mínima es 22,2 . b 0 casillas $(0 \%)$ tienen frecuencias esperadas menores que 5 . La frecuencia de casilla esperada mínima es 19,5. c 0 casillas $(0 \%)$ tienen frecuencias esperadas menores que 5 . La frecuencia de casilla esperada mínima es 22,5 . d 0 casillas $(0 \%)$ tienen frecuencias esperadas menores que 5 . La frecuencia de casilla esperada mínima es 18,5. Fuente: elaboración propia a partir de SPSS v.17.

mayores discrepancias positivas del resultado global frente al resultado neto, seguidos por los de tamaño mediano, y ya a una mayor distancia por los pequeños.

Sin embargo, para el resto de años, salvo para 2003 , la tendencia es la con- traria, en especial para los años 2001 y 2002, en los que se registran las máximas diferencias entre los distintos niveles por tamaño teniendo en cuenta los casos que exceden de la mediana. Los grupos empresariales pequeños, en conjunto, pre- 
sentan para esos años mayores discrepancias positivas que los medianos y grandes con respecto al impacto relativo del resultado global frente al resultado neto.

Del mismo modo, de acuerdo con el Test de la Mediana, según se recoge en la Tabla 15, las importantes diferencias entre los distintos niveles por tamaño de los casos que exceden de la misma o no la alcanzan, llevan a $p<0,05$; y por ello a unos resultados significativos para los años 1999, 2001, 2002 y 2004, con lo que según este test se confirman los resultados alcanzados con la prueba de Kruskal-Wallis, y aún se detectan para un año más, en concreto para 2004, diferencias significativas entre los diferentes niveles asociados a la dimensión empresarial.

De acuerdo con las anteriores pruebas a priori se sabe que existen dife- rencias estadísticamente significativas esos años, pero no se conoce entre qué niveles por tamaño se presentan, por lo que se hace necesario practicar en principio como test post hoc la prueba de la $U$ de Mann-Whitney (Tablas 16 a 21), evidentemente solo para los años en los que las pruebas de Kruskal-Wallis y de la Mediana detectaron diferencias significativas.

Al observar las Tablas 16,18 y 20 se encuentran bastantes diferencias notables en los promedios de los rangos, que conducen a $p<0,05$; y por ello a diferencias significativas entre los grupos empresariales pequeños y medianos para el año 2002 (Tabla 17), entre los pequeños y grandes para 1999, 2001 y 2002 (Tabla 19), y por último entre los medianos y grandes para 2001 (Tabla 21).

Tabla 16

Rangos de la Prueba U de Mann-Whitney*

\begin{tabular}{ccccc}
\hline & Tamaño & N & Rango promedio & Suma de rangos \\
\hline \multirow{3}{*}{ CINI1999 } & Pequeño & 45 & 42,20 & $1.899,00$ \\
& Mediano & 46 & 49,72 & $2.287,00$ \\
& Total & 91 & & \\
\hline \multirow{3}{*}{ CINI2001 } & Pequeño & 45 & 48,41 & $2.178,50$ \\
& Mediano & 46 & 43,64 & $2.007,50$ \\
& Total & 91 & & $2.640,50$ \\
CINI2002 & Pequeño & 45 & 58,68 & $1.545,50$ \\
& Mediano & 46 & 33,60 & $2.311,00$ \\
& Total & 91 & & $1.875,00$ \\
CINI2004 & Pequeño & 45 & 51,36 & \\
& Mediano & 46 & 40,76 & \\
& Total & 91 & & \\
\hline
\end{tabular}

*Comparación por tamaño pequeño/mediano.

Fuente: elaboración propia, a partir de SPSS v.17. 
Tabla 17

Estadísticos de contraste de la Prueba U de Mann-Whitney*

\begin{tabular}{lrrrr}
\hline & CINI1999 & CINI2001 & CINI2002 & CINI2004 \\
\hline U de Mann-Whitney & 864,000 & 926,500 & 464,500 & 794,000 \\
W de Wilcoxon & $1.899,000$ & $2.007,500$ & $1.545,500$ & $1.875,000$ \\
Z & $-1,362$ & $-0,865$ & $-4,539$ & $-1,917$ \\
Sig. asintót. (bilateral) & 0,173 & 0,387 & 0,000 & 0,055 \\
\hline
\end{tabular}

*Comparación por tamaño pequeño/mediano.

Fuente: elaboración propia, a partir de SPSS v.17.

Tabla 18

Rangos de la Prueba U de Mann-Whitney*

\begin{tabular}{ccccc}
\hline & Tamaño & N & Rango promedio & Suma de rangos \\
\hline \multirow{3}{*}{ CINI1999 } & Pequeño & 45 & 38,56 & $1.735,00$ \\
& Grande & 45 & 52,44 & $2.360,00$ \\
& Total & 90 & & \\
\hline \multirow{3}{*}{ CINI2001 } & Pequeño & 45 & 56,04 & $2.522,00$ \\
& Grande & 45 & 34,96 & $1.573,00$ \\
& Total & 90 & & $2.669,00$ \\
& Pequeño & 45 & 59,31 & $1.426,00$ \\
\hline \multirow{3}{*}{ CINI2002 } & Grande & 45 & 31,69 & $2.207,00$ \\
& Total & 90 & & $1.888,00$ \\
& Pequeño & 45 & 49,04 & \\
& Grande & 45 & 41,96 & \\
\hline
\end{tabular}

${ }^{*}$ Comparación por tamaño pequeño/grande.

Fuente: elaboración propia, a partir de SPSS v.17.

$5 \quad$ En Sieger y Castellan (1988: 128), una obra de referencia a nivel internacional en herramientas de contraste no paramétricas, se señala que las pruebas de la $U$ de Mann-Whitney y W de Wilcoxon fueron propuestas independientemente, entre otros, por estos autores, y esencialmente representan lo mismo. Se ha revelado la información tal como aparece en los resultados de SPSS v.17. Por otra parte, se recuerda que esta prueba de Wilcoxon hace referencia a la suma de rangos, y no a la suma de rangos con signo con una metodología evidentemente diferente a ésta. 


\section{Tabla 19}

Estadísticos de contraste de la Prueba U de Mann-Whitney*

\begin{tabular}{lrrrr}
\hline & CINI1999 & CINI2001 & CINI2002 & \multicolumn{1}{c}{ CINI2004 } \\
\hline U de Mann-Whitney & 700,000 & 538,000 & 391,000 & 853,000 \\
W de Wilcoxon & $1.735,000$ & $1.573,000$ & $1.426,000$ & $1.888,000$ \\
Z & $-2,527$ & $-3,842$ & $-5,025$ & $-1,290$ \\
Sig. asintót. (bilateral) & 0,012 & 0,000 & 0,000 & 0,197 \\
\hline
\end{tabular}

${ }^{*}$ Comparación por tamaño pequeño/grande.

Fuente: elaboración propia, a partir de SPSS v.17.

\section{Tabla 20}

Rangos de la Prueba U de Mann-Whitney*

\begin{tabular}{ccccc}
\hline & Tamaño & $N^{\circ}$ & Rango promedio & Suma de rangos \\
\hline \multirow{3}{*}{ CINI1999 } & Mediano & 45 & 41,41 & $1.905,00$ \\
& Grande & 46 & 50,69 & $2.281,00$ \\
& Total & 91 & & \\
\hline \multirow{3}{*}{ CINI2001 } & Mediano & 45 & 54,96 & $2.528,00$ \\
& Grande & 46 & 36,84 & $1.658,00$ \\
& Total & 91 & & $2.167,00$ \\
& Mediano & 45 & 47,11 & $2.019,00$ \\
\hline \multirow{2}{*}{ CINI2004 } & Grande & 46 & 44,87 & $2.043,00$ \\
& Total & 91 & & $2.143,00$ \\
& Mediano & 45 & 44,41 & \\
\hline
\end{tabular}

*Comparación por tamaño mediano/grande.

Fuente: elaboración propia, a partir de SPSS v.17.

Tabla 21

Estadísticos de contraste de la Prueba U de Mann-Whitney*

\begin{tabular}{lrrrr}
\hline & CINI1999 & CINI2001 & CINI2002 & CINI2004 \\
\hline U de Mann-Whitney & 824,000 & 623,000 & 984,000 & 962,000 \\
W de Wilcoxon & $1.905,000$ & $1.658,000$ & $2.019,000$ & $2.043,000$ \\
Z & $-1,675$ & $-3,270$ & $-0,405$ & $-0,058$ \\
Sig. asintót. (bilateral) & 0,094 & 0,001 & 0,686 & 0,562 \\
\hline
\end{tabular}

*Comparación por tamaño mediano/grande.

Fuente: elaboración propia, a partir de SPSS v.17. 
Asimismo, como las variables no se distribuyen de forma similar entre los tres niveles por tamaño, tal como quedó justificado, se ha considerado adecuado practicar el test de Kolmogorov-Smirnov para dos muestras para complementar y dar refuerzo a las anteriores revelaciones, con los resultados que se recogen en las Tablas 22 a 24.

Al observar las mismas se encuentra $p<0,05$; y por ello diferencias significativas entre los grupos empresariales pequeños y medianos para 2002 y 2004 (Tabla 22), entre los pequeños y grandes en 1999, 2001, 2002 y 2004 (Tabla 23), y por último entre los medianos y grandes para 2001 (Tabla 24), lo que además de confirmar las diferencias detectadas por la $U$ de Mann-Whitney, se revelan otras nuevas, las correspondientes a 2004.

Al tomar los resultados conjuntos de las pruebas de la $U$ de Mann-Whitney y de Kolmogorov-Smirnov para dos muestras, se confirman los años 2001 y 2002 con diferencias significativas, que como se ha visto son los peores años del período estudiado, con una clara recesión económica y fuertes caídas bursátiles, y es precisamente donde la U de Ma$\mathrm{nn}$-Whitney detecta las medias de rangos más favorables a los grupos empresariales pequeños frente a las grandes. Con todo ello, se apunta a que el resultado global en los malos años económicos y

Tabla 22

Estadísticos de contraste de Kolmogorov-Smirnov para dos muestras*

\begin{tabular}{lccccc}
\hline & & CINI1999 & CINI2001 & CINI2002 & CINI2004 \\
\hline & Absoluta & 0,277 & 0,231 & 0,563 & 0,363 \\
Diferencias más extremas & Positiva & 0,067 & 0,231 & 0,563 & 0,363 \\
& Negativa & $-0,277$ & $-0,125$ & 0,000 & $-0,042$ \\
\hline Z de Kolmogorov-Smirnov & & 1,320 & 1,104 & 2,687 & 1,733 \\
Sig. asintót. (bilateral) & & 0,061 & 0,175 & 0,000 & 0,005 \\
\hline
\end{tabular}

*Comparación por tamaño pequeño/mediano.

Fuente: elaboración propia, a partir de SPSS v.17.

Tabla 23

Estadísticos de contraste de Kolmogorov-Smirnov para dos muestras*

\begin{tabular}{lccccc}
\hline & & CINI1999 & CINI2001 & CINI2002 & CINI2004 \\
\hline & Absoluta & 0,400 & 0,489 & 0,622 & 0,356 \\
Diferencias más extremas & Positiva & 0,400 & 0,000 & 0,000 & 0,133 \\
& Negativa & $-0,022$ & $-0,489$ & $-0,622$ & $-0,356$ \\
\hline Z de Kolmogorov-Smirnov & & 1,897 & 2,319 & 2,951 & 1,687 \\
Sig. asintót. (bilateral) & & 0,001 & 0,000 & 0,000 & 0,007 \\
\hline
\end{tabular}

*Comparación por tamaño pequeño/grande

Fuente: elaboración propia, a partir de SPSS v.17. 


\section{Tabla 24}

Estadísticos de contraste de Kolmogorov-Smirnov para dos muestras*

\begin{tabular}{lccccc}
\hline & & CINI1999 & CINI2001 & CINI2002 & CINI2004 \\
\hline & Absoluta & 0,230 & 0,425 & 0,137 & 0,137 \\
Diferencias más extremas & Positiva & 0,230 & 0,022 & 0,096 & 0,137 \\
& Negativa & $-0,048$ & $-0,425$ & $-0,137$ & $-0,042$ \\
\hline Z de Kolmogorov-Smirnov & & 1,097 & 2,028 & 0,652 & 0,654 \\
Sig. asintót. (bilateral) & & 0,180 & 0,001 & 0,789 & 0,785 \\
\hline
\end{tabular}

${ }^{*}$ Comparación por tamaño mediano/grande.

Fuente: elaboración propia, a partir de SPSS v.17.

bursátiles, o lo que se puede denominar el efecto "crisis", tiende a castigar mucho más los resultados de los grupos empresariales grandes y medianos que los de los pequeños.

Asimismo, el año 1999 fue el mejor ciclo bursátil de todo el período, y es precisamente en el mismo cuando de acuerdo con la U de Mann-Whitney se encuentra la diferencia en la media de rangos más favorable a los grupos empresariales grandes frente a los pequeños, que condujo evidentemente a pruebas significativas. Así, se está apuntando a que en los buenos años en la economía en general y en las bolsas, o lo que se puede denominar con el efecto "expansión", el resultado global tiende a mejorar las cuentas de resultados de los grupos empresariales grandes con respecto a las de los pequeños.

En definitiva, el conjunto de revelaciones que se han hecho en este apartado del trabajo de investigación, no hacen sino encontrar explicación a la actitud reticente de las empresas, en especial las grandes corporaciones del sector financiero, contrarias a la adopción del resultado global, en particular, a lo regulado al respecto en la controvertida NIC 39 , o la fuerte oposición a la aprobación del SFAS 130, por temor a que sus cuentas de resultados se viesen lastradas.

Con todo ello, dada la naturaleza de los ítems que incorpora el resultado global en los resultados empresariales, a través de las evidencias empíricas encontradas se puede deducir para el período de estudio una mayor exposición al riesgo de mercado de los grupos empresariales grandes y medianos frente a los pequeños, debido a que los primeros realizaron mayores dotaciones relativas a la cobertura del fondo de pensiones de los empleados, tuvieron más posiciones relativas en moneda extranjera; $y$ en especial, para los bancos y compañías de seguros su cartera de inversiones financieras disponibles para la venta fue también superior en términos relativos.

Si a este mayor negocio relativo de las grandes corporaciones frente a las más pequeñas en partidas asociadas al resultado global, se une el hecho del auge económico y bursátil de 1999, la crisis económica y fuerte caída de las cotizaciones bursátiles registradas en los años 2001 y 2002, junto con la posterior 
recuperación en los años 2003 y 2004, y la notable apreciación del euro frente al dólar USA en los años 2002, 2003 y 2004; se pueden explicar una buena parte de las acusadas diferencias que se han revelado, en particular, para las grandes corporaciones frente a las pequeñas, que explican los temores a los que se han aludido, pero se entiende que no son justificables si se quiere que el resultado contable se aproxime cada vez más a la realidad de los mercados.

\section{Conclusiones}

El resultado global, conocido en el mundo anglosajón como comprehensive income, está cobrando cada vez más fuerza como referente del resultado empresarial frente al más tradicional resultado neto, mucho menos vinculado a la realidad de los mercados.

Ello es así porque tanto el FASB como el IASB, los dos organismos reguladores de la Contabilidad de más influencia a nivel mundial, vienen exigiendo a las empresas en sus respectivos cuerpos normativos la revelación del mismo, fundamentalmente para atender las necesidades de los usuarios de la información financiera, en particular, las de los inversores, considerados en el Marco Conceptual como usuarios de referencia.

Además, los modelos contables de la mayor parte de países del mundo ya han adaptado o están en proceso de adaptación de su normativa contable interna a las NIIF del IASB, como ha ocurrido en muchos países latinoamericanos, cual es el caso de Venezuela con la emisión de los Boletines de Aplicación de las normas del IASB, emitidos por la Federa- ción de Colegios de Contadores Públicos, o en la Unión Europea con la aprobación de los Reglamentos de adopción de dichas normas.

En consecuencia, se está asistiendo ahora a los inicios del siglo XXI, entre otras novedades importantes que incorporan las NIIF del IASB, a la adopción a escala universal del resultado global, un nuevo concepto de resultado empresarial, en particular, para los países cuyos modelos contables han estado fuera del área de influencia de la Contabilidad anglosajona, como pueden ser los países de Latinoamérica o los de la Europa Continental.

De este modo, las empresas habrán de revelar en sus Cuentas de Resultados el resultado neto, más ligado al modelo contable tradicional cimentado en el coste histórico y en el principio de realización; y han de informar también del resultado global, que además de integrar al primero, da entrada en las valoraciones contables a valores de mercado, como puede ser el valor razonable en la actualidad, con el consiguiente abandono del principio de realización al reconocerse en resultados tanto potencialidades de beneficios como de pérdidas.

En el marco de estas tendencias en la información financiera internacional, se ha evaluado empíricamente cómo impacta el resultado global en las empresas atendiendo a su dimensión, a partir de una muestra de 136 grupos empresariales europeos cotizados en NYSE y NAS$D A Q$, de acuerdo con la correspondiente información revelada en las reconciliaciones con los US GAAP en los 20-F al presentar sus cuentas a la SEC. Como metodología de contraste se ha empleado un 
conjunto de herramientas no paramétricas al estar los datos muy alejados de la normalidad.

Aunque se haya utilizado el resultado global de acuerdo con US GAAP, y existan algunas diferencias con respecto al mismo resultado a revelar según IASB GAAP, se considera que este estudio puede representar un anticipo de lo que ya está siendo la aplicación del resultado global a escala mundial según este último cuerpo normativo. Además, a partir de este estudio se suscita el desarrollo de futuros trabajos, utilizando muestras y períodos diferentes, como puede ser el caso de las cotizadas norteamericanas con los US GAAP, o de países latinoamericanos y europeos en la primera andadura de la aplicación de las normas del IASB.

De acuerdo con los estadísticos descriptivos de las variables que sirvieron de referencia para practicar los contrastes, se evidencia un impacto relativo del resultado global frente al resultado neto, que se puede calificar de espectacular, ya que para el conjunto de los 136 grupos empresariales integrantes de la muestra y los seis años estudiados, la adopción del resultado global frente al más tradicional resultado neto ha hecho empeorar los resultados más de un veinticinco por ciento por término medio.

Este impacto negativo tan acusado se debe a los tipos de cambio desfavorables al calcular las diferencias de conversión de los activos y pasivos de las filiales en el proceso de consolidación, a las fuertes caídas bursátiles de principios de los dos mil con incidencia muy negativa en la valoración de los activos financieros clasificados como disponibles para la venta a efectos de su valoración, sin olvidar la notable incidencia negativa del ajuste por la cobertura mínima del fondo de pensiones de los empleados. Todo ello, prueba una mayor sensibilidad o conexión del resultado global con la realidad de los mercados que el resultado neto.

Asimismo, al entrar a particularizar el impacto relativo del resultado global frente al resultado neto atendiendo a la dimensión de los grupos empresariales, de acuerdo con los estadísticos descriptivos se encuentran notables diferencias entre los grupos de pequeña, mediana y gran dimensión.

Así, por término medio para los seis años estudiados, los de pequeña dimensión mejoran sus resultados en más de un ocho por ciento al adoptar el resultado global frente al resultado neto. Pero en este sentido, los de mediana dimensión empeoran sus resultados de forma muy acusada, al hacerlo en más de un veintiséis por ciento, y los de gran dimensión sufren unos efectos negativos que se pueden calificar de espectaculares, al hacerlo en más de un cincuenta y siete por ciento.

Este diferente comportamiento de los grupos empresariales atendiendo a su dimensión que ya señalan de forma muy clara los estadísticos descriptivos, se confirma al practicar los contrastes con las herramientas no paramétricas.

De este modo, se han hallado diferencias estadísticamente significativas con respecto al impacto relativo del resultado global frente al resultado neto, entre los grupos empresariales pequeños y medianos los años 2002 y 2004 , entre los pequeños y grandes los años 1999, 2001, 2002 y 2004, y por último entre las medianos y grandes el año 2001. 
Además, si se consideran los años en los que se registran dichas diferencias significativas, el resultado global en los malos años económicos y bursátiles, o lo que se puede denominar el efecto "crisis", tiende a castigar mucho más los resultados de las grandes y medianas corporaciones que los de las pequeñas. Y en sentido contrario, en los buenos años en la economía en general y en las bolsas, o lo que se puede denominar con el efecto "expansión", el resultado global tiende a mejorar las cuentas de resultados de las grandes corporaciones con respecto a las de las pequeñas. No obstante, tal como se ha indicado, resultaría de interés desarrollar futuros estudios con muestras, periodos y GAAP diferentes que permitieran encontrar más evidencias empíricas al respecto.

En cualquier caso, el conjunto de hallazgos empíricos de este trabajo de investigación, no hacen sino encontrar explicación a la actitud reticente de las empresas, en especial las grandes corporaciones del sector financiero, contrarias a la adopción del resultado global, en particular, a lo regulado al respecto en la controvertida NIC 39, o la fuerte oposición a la aprobación del SFAS 130, por temor a que sus cuentas de resultados se viesen perjudicadas, en particular, la imagen de estabilidad de su beneficio a lo largo de una serie de ejercicios económicos.

No obstante, mucho más allá de las políticas contables que puedan interesar a determinadas corporaciones, tendentes a alisar el resultado empresarial, se considera que si los precios de los títulos y los tipos de cambio fluctúan, la Contabilidad, y en particular, el resultado empre- sarial, no ha de dar una imagen de estabilidad, sino ser reflejo de la realidad.

Como reflexión final, se ha de tener en cuenta que la adopción del resultado global representa uno de los elementos esenciales en la transición desde un modelo contable arraigado en el coste histórico a lo largo del siglo XX, hacia otro en el que coexiste dicho criterio con los valores corrientes como el valor razonable en la actualidad; lo que hace necesario un cambio de mentalidad en la forma de concebir el resultado empresarial para los que tienen responsabilidades gerenciales, los investigadores y el resto de usuarios interesados en la información financiera.

\section{Referencias bibliográficas}

Alexander, Sidney S. (1950), "Income Measurement in a Dynamic Economy", en Five Monographs on Business Income, Study Group on Business Income, American Institute of Accountants (AIA), New York, pp. 1-95.

Beale, Bob y Howard Davey (2000), "The Nature and Origins of Comprehensive Income", en S. B. Dahiya (ed.), The Current State of Business Disciplines, SpellBound Publications Ltd., New Delhi, Vol. 1, pp. 81-100.

Bhamornsiri, Sak y Casper Wiggins (2001), Comprehensive Income Disclosures, The CPA Journal, October, pp. 5456.

Brief, Richard P. y Kenneth V. Peasnell (1996), Clean Surplus: A link Between Accounting and Finance, Garland Publishing, Inc., New York and London.

Dhaliwal, Dan.; K. R. Subramanyam y Robert Trezevant (1999), Is Comprehensive Income Superior to Net Income as a Measure of Firm Performance, Jour- 
nal of Accounting and Economics, Vol. 26, pp. 43-67.

Edwards, Edgar O. y Philip W. Bell (1961), The Theory and Measurement of Business Income, University of California Press, Seven Printing, 1973, Berkeley, Los Angeles, London.

Feltham, Gerald A. y James A. Ohlson (1995), Valuation and Clean Surplus Accounting for Operating and Financial Activities, Contemporary Accounting Research, Vol. 12, Spring, pp. 689-731.

Federación de Contadores Públicos de la República Bolivariana de Venezuela (2010), Boletín de aplicación de VEN-NIF N$^{\circ} 0$ (BA VEN-NIF 0), disponible en http://www.fccpv.org/ cont3/ data/files/BA_VEN-NIF_0_VERSION _4.pdf [acceso el 4 de junio de 2010].

Financial Accounting Standards Board, FASB (1997), SFAS 130, "Reporting Comprehensive Income", FASB, disponible en http://www.fasb.org, [acceso el 3 de mayo de 2010].

Statement of Financial Accounting Concepts, SFAC 3 (1980), "Elements of Financial Statements of Business Enterprises", disponible en http:// www.fasb.org, [acceso el 3 mayo de 2010].

Statement of Financial Accounting Concepts, SFAC 6 (1985), "Elements of Financial Statements - a replacement of FASB Concepts Statement No. 3 (incorporating an amendment of FASB Concepts Statement No. 2)", disponible en http://www.fasb.org, [acceso el 3 mayo de 2010].

Hicks, John Richard (1939), Value and Capital, Oxford University Press, London.

Hirst, D. Erik, y Patrick E. Hopkins (1998), Comprehensive Income Reporting and Analysts' Valuation Judgements, Journal of Accounting Research, Vol. 36, Supplement, pp. 47- 75.
Hodder, Leslie D., Patrick E. Hopkins, y James M. Wahlen (2006), Risk-Relevance of Fair-Value Income Measures for Commercial Banks, The Accounting Review, Vol. 81, n² 2, pp. 337-375.

Hunton, James E., Robert Libby y Chery L. Mazza (2006), Financial Reporting Transparency and Earnings Management, The Accounting Review, Vol. 81, n 1, pp. 135-157.

International Accounting Standards Board (2008), International Financial Reporting Standards, Bond Volume, 2008, IASB-IASC, London:

International Accounting Standards Board (2003) NIC 39, Financial Instruments: Recognition and Measurement.

International Accounting Standards Board (2007) NIC 1, Presentation of Financial Statements.

International Accounting Standards Board (2003), NIC 1 "Presentation of Financial Statements", IASB, London.

International Accounting Standards Board (1997), NIC 1 "Presentation of Financial Statements”, IASB, London.

Linsmeier, Thomas J; John Gribble; Ross Jennings; Mark H. Lang; Stephen H. Penman; Katty R. Petroni; D. Shores; John H. Smith y Terry D. Warfield (1997), An Issues Paper on Comprehensive Income, American Accounting Association's Financial Accounting Standards Committee, Accounting Horizons, Vol. 11, $n^{\circ} 2$, pp. 120126.

Luecke, Randall W. y David T. Meeting (1998), How Companies Report Income, Journal of Accountancy, May, pp. 45-52.

MacNeal, Kenneth (1939), Truth in Accounting, University of Pennsylvania Press, Philadelphia. 
Maines, Laureen A. y Linda S. McDaniel (2000), Effects of ComprehensiveIncome Characteristics on Nonprofessional Investors' Judgements: The Role of Financial-Statement Presentation Format, The Accounting Review, Vol. 75, No. 2, pp. 179-207.

Mattessich, Richard (2002), Teoría del Excedente Limpio y su Evolución: Revisión y Perspectivas Recientes, Energeia: Revista Internacional de Filosofía y Epistemología, Vol. 1, $n^{\circ} 2$, Universidad de Ciencias Empresariales y Sociales de Buenos Aires, Spanish Translation, pp. 49-79.

Moonitz, Maurice (1961), The Basic Postulates of Accounting, Accounting Research Study No. 1, American Institute of Certified Public Accountants (AICPA), New York.

Mora Enguídanos, Araceli (2004), La normativa internacional sobre instrumentos financieros: un debate sin resolver, Accésit del X Premio AECA para Artículos sobre Contabilidad y Administración de Empresas y del Sector Público, Revista de AECA, $n^{\circ}$ 67, pp. 8-10.

National Association of Securities Dealers Automated Quotations, NASDAQ (2004), NASDQ International Companies, Europe, disponible en http://www.nasdaq.com/asp/NonUsOutput. asp?page=B\&region= europe, [acceso el día 31 de diciembre de 2004].

New York Stock Exchange, NYSE (2004), Non-U.S. Listed Company Directory, Europe, listado disponible en http://www.nyse.com/international/nonuslisted/int_listed.html, [acceso el día 31 de diciembre de 2004].

O'Hanlon, John F. y Peter F. Pope (1999), The Value-Relevance of UK Dirty Surplus Accounting Flows, British Accounting Review, Vol. 31, pp. 459-482.
Pandit, Ganesh M.; Allen Rubenfield y Jeffrey J. Phillips (2006), Current NASDAQ Corporation Methods of Reporting Comprehensive Income, Mid-merican Journal of Business, Spring, 21,1. pp. 13-19.

Siegel, Sidney y N. John Castellan Jr. (1988), Non Parametric Statistics for the Behavioral Sciences, MacGraw-Hill International Sciences, Second Edition, International Edition.

Sousa Fernández, Francisco (2008), Impacto del comprehensive income frente al net income en los grupos empresariales españoles cotizados en la Bolsa de Madrid, Revista de Contabilidad y Tributación, CEF, n³09, pp.165214.

Sousa Fernández, Francisco (2009a), Fundamentos Conceptuales del Resultado Global -Conceptual Foundations of Comprehensive Income-, Revista de Contabilidad -Spanish Accounting Review-, Vol. 12, n² 2, pp. 215-252.

Sousa Fernández, Francisco (2009b), Strong Impact Of Comprehensive Income on European Groups Listed in American Markets, International Business \& Economics Research Journal, Vol, 8, No. 3, pp. 117-129.

Sousa Fernández, Francisco y María Mercedes Carro Arana (2009a), Impacto del comprehensive income frente al net income en la estabilidad del beneficio del BSCH y BBVA según US GAAP (1998-2007), Universia Business Review, $n^{\circ} 21$, pp. 118-133.

Sousa Fernández, Francisco y María Mercedes Carro Arana (2009b), Empirical Evaluation Of The Overall Impact And By Industries Of Comprehensive Income On European Groups Listed On NYSE and NASDAQ, International Business \& Economics Research Journal, Vol. 8, No. 4, p. 91-112. 
Sousa Fernández, Francisco y María Mercedes Carro Arana (2009c), Empirical Analysis Of The Impact Of Comprehensive Income On Basic Earnings Per Share For Spanish Companies Listed On Madrid Stock Exchange, International Business \& Economics Research Journal, Vol. 8, No. 6, p. 7-23.

Sprouse, Robert T. y Maurice Moonitz (1962),

A Tentative Set of Broad Accounting Principles for Business Enterprises, Accounting Research Study No. 3, American Institute of Certified Public Accountants (AICPA), New York.
Statistical Package for the Social Sciences, SPSS (2009), Programa SPSS, versión 17.0, SPSS Inc.

U.S. Securities and Exchange Commission, SEC (2004), the Electronic Data Gathering, Analysis, and Retrieval system (EDGAR), Base de Datos EDGAR de la SEC, Form 20-F, de los años que estuviesen disponibles para los grupos del continente europeo listados en NYSE y el NASDAQ, disponible en http://www.sec.gov/edgar/ searchedgar/companysearch.html [acceso desde julio de 2004 a diciembre de 2004]. 